NIKHEF-99-002

SPhT-T99/004

\title{
QCD Corrections to Four-jet Production and Three-jet Structure in $e^{+} e^{-}$annihilation
}

\author{
Stefan Weinzierl ${ }^{1, a}$ and David A. Kosower ${ }^{2, b}$ \\ 1 NIKHEF, P.O. Box 41882, NL - 1009 DB Amsterdam, The Netherlands \\ 2 Service de Physique Théorique, Centre d'Etudes de Saclay, \\ F-91191 Gif-sur-Yvette Cedex, France
}

\begin{abstract}
We report on the general purpose numerical program Mercutio, which can be used to calculate any infrared safe four-jet quantity in electron-positron annihilation at next-to-leading order. The program is based on the dipole formalism and uses a remapping of phase-space in order to improve the efficiency of the Monte Carlo integration. Numerical results are given for the four-jet fraction and the $D$ parameter. These results are compared with already existing ones in the literature and serve as a cross-check. The program can also be used to investigate the internal structure of three-jet events at NLO. We give results for previously uncalculated observables: the jet broadening variable and the softest-jet explanarity.
\end{abstract}

${ }^{a}$ email address : stefanw@nikhef.nl

${ }^{b}$ email address : kosower@spht.saclay.cea.fr 


\section{Introduction}

Electron-positron annihilation provides a clean environment for studying jet physics, as strong interaction effects occur only in the final state. We thus avoid the complications of parton distribution functions and their associated uncertainties. Perturbative QCD corrections to jet variables can nonetheless be sizeable. The most recent addition to the list of known one-loop amplitudes are those for $e^{+} e^{-} \rightarrow 4$ partons, which have been calculated by two groups independently. Our group obtained analytic expressions for the amplitudes [四] by calculating helicity amplitudes using the cut technique. The Durham group performed a conventional calculation and provided computer code [2]. The two sets of expressions have been shown to agree numerically. Here we report on the implementation of the one-loop amplitudes into a numerical program MERCUTIO, which can be used to calculate any infrared safe four-, three- or two-jet quantity at next-to-leading order (and five-jet quantities at leading order). It is similar to Menlo Parc [3], Debrecen [4] and EERAD2 [5]. The main difference among the programs is the technique used for the cancellation of real and virtual singularities. MENLO PARC uses the subtraction method of ref. [6], DEBRECEn the dipole method [7], while EERAD2 uses a hybrid of the phasespace slicing [8, 9] and subtraction methods. MERCUTIO also uses the dipole formalism, but with a remapping of phase space. A naive implementation of the dipole formalism will give large statistical errors when performing a Monte Carlo integration over the real corrections with dipole factors subtracted. In order to improve the efficiency of the Monte Carlo integration we remap the phase space in order to make the integrand more flat.

With the resulting numerical program, we first compute results for several observables studied previously in the literature. We present results for the four-jet fraction and the $D$-parameter, which agree with the results from the other programs. We also present results for quantities describing the internal structure of three-jet events. We give numerical results for the jet broadening and the softest-jet explanarity.

The paper is organized as follows: in section 2 we explain the implementation of the dipole formalism and the remapping of phase space. Section 3 deals with technical details concerning various dimensional regularization schemes. Section 4 is devoted to phenomenology, and contains our numerical results. The conclusions are given in section 5 .

\section{Cancellation of Infrared Divergences}

The NLO cross section receives contributions both from virtual corrections and from real emission. For $e^{+} e^{-} \rightarrow 4$ jets the virtual part consists of the interference terms between the tree amplitudes and the one-loop amplitudes for $e^{+} e^{-} \rightarrow q g g \bar{q}$ and $e^{+} e^{-} \rightarrow q \bar{q} q^{\prime} \bar{q}^{\prime}$. The real emission part consists of the squared tree-level amplitudes with one additional parton, namely $e^{+} e^{-} \rightarrow q g g g \bar{q}$ and $e^{+} e^{-} \rightarrow q \bar{q} q^{\prime} \bar{q}^{\prime} g$, which were computed long ago 10. Both the virtual and the real emission contributions contain infrared divergences, arising when one particle becomes soft or two particles collinear. Only the sum of the virtual corrections and the real emission part is finite. Because the virtual part is integrated over $n$-particle phase-space, whereas the real emission part is integrated over the $(n+1)$-particle phase 
space, it is not possible to integrate the divergent contributions numerically and to cancel the divergences after the (numerical) integration. The cancellation of infrared singularities has to be performed before any numerical integration is done. There are basically two approaches to overcome this obstacle: phase-space slicing [9] and the subtraction method. (Within the subtraction method there are two variants: the subtraction method by Frixione, Kunszt and Signer [6] and the dipole formalism of Catani and Seymour [7].) As mentioned above, MERcutio uses the dipole formalism in order to handle infrared divergences.

Throughout this paper the conventional normalization of the color matrices is used: $\operatorname{Tr} T^{a} T^{b}=\frac{1}{2} \delta^{a b}$.

\subsection{The dipole formalism}

The dipole formalism [7] is based on the subtraction method. The NLO cross section is written as

$$
\begin{aligned}
\sigma^{N L O} & =\int_{n+1} d \sigma^{R}+\int_{n} d \sigma^{V} \\
& =\int_{n+1}\left(d \sigma^{R}-d \sigma^{A}\right)+\int_{n}\left(d \sigma^{V}+\int_{1} d \sigma^{A}\right),
\end{aligned}
$$

where in the last line an approximation term $d \sigma^{A}$ has been added and subtracted. The approximation $d \sigma^{A}$ has to be a proper approximation of $d \sigma^{R}$ such as to have the same pointwise singular behaviour in $D$ dimensions as $d \sigma^{R}$ itself. Further $d \sigma^{A}$ has to be analytically integrable in $D$ dimensions over the one-parton subspace leading to soft and collinear divergences. According to Catani and Seymour the approximation term is written as the $n$-parton Born term $d \sigma^{B}$ times the dipole factors,

$$
d \sigma^{A}=\sum_{\text {dipoles }} d \sigma^{B} \otimes d V_{\text {dipole }}
$$

The integration over the $(n+1)$-parton phase space yields

$$
\int_{n+1} d \sigma^{A}=\sum_{\text {dipoles }} \int_{n} d \sigma^{B} \otimes \int_{1} d V_{\text {dipole }}=\int_{n}\left[d \sigma^{B} \otimes I\right],
$$

where the universal factor $I$ is defined by

$$
I=\sum_{\text {dipoles } 1} \int_{1} d V_{\text {dipoles }}
$$

For the explicit formulæ we refer to the paper of Catani and Seymour [7]. Here, we discuss the implementation of the dipole formalism when using helicity amplitudes. Let $A^{\mu}$ denote the $n$-parton amplitude, where the polarization vector $\varepsilon_{\mu}$ of the emitter gluon has been amputated. Using

$$
\left(\varepsilon_{+}^{\mu}\right)^{*} \varepsilon_{+}^{\nu}+\left(\varepsilon_{-}^{\mu}\right)^{*} \varepsilon_{-}^{\nu}=-g^{\mu \nu}+\frac{\tilde{p}_{i j}^{\mu} q^{\nu}+\tilde{p}_{i j}^{\nu} q^{\mu}}{\tilde{p}_{i j} \cdot q},
$$


where $q$ is an arbitrary reference momentum, we obtain

$$
\left(A^{\mu}\right)^{*}\left(-g_{\mu \nu}\right) A^{\nu}=A_{+}^{*} A_{+}+A_{-}^{*} A_{-}
$$

where $A_{ \pm}$denotes the helicity amplitude where the emitter gluon has positive or negative

helicity. The dependence on the reference momentum $q$ drops out, since $\left(\tilde{p}_{i j}\right)_{\mu} A^{\mu}=0$ (gauge invariance), $q_{\mu} \varepsilon^{\mu}=0$ (property of the polarization vectors) and $\left(\tilde{p}_{i j}\right)^{2}=0$ (the gluon is on mass-shell). For the spin correlation we obtain

$$
\left(A_{\mu}\right)^{*}\left(\tilde{z}_{i} p_{i}^{\mu}-\tilde{z}_{j}^{\mu} p_{j}^{\mu}\right)\left(\tilde{z}_{i} p_{i}^{\nu}-\tilde{z}_{j}^{\nu} p_{j}^{\nu}\right) A_{\nu}=\left|E A_{+}+E^{*} A_{-}\right|^{2},
$$

where

$$
E=\frac{\left\langle q+\left|\tilde{z}_{i} p_{i}-\tilde{z}_{j} p_{j}\right| \tilde{p}_{i j}+\right\rangle}{\sqrt{2}\left[q \tilde{p}_{i j}\right]}
$$

Using the fact that the spin correlation tensor is orthogonal to $\tilde{p}_{i j}$ one shows again that the dependence on the reference momentum drops out. As reference momentum one may choose $q=p_{j}$, in that case $E$ reduces to

$$
E=\frac{\tilde{z}_{i}\left[p_{j} p_{i}\right]\left\langle p_{i} \tilde{p}_{i j}\right\rangle}{\sqrt{2}\left[p_{j} \tilde{p}_{i j}\right]}
$$

\subsection{Improving the Dipole Formalism}

Within the dipole formalism we have to evaluate the terms

$$
\int_{n}\left(d \sigma^{V}+d \sigma^{B} \otimes I\right) \text { and } \int_{n+1} d \sigma^{R}-d \sigma^{A} .
$$

The first term is most efficiently integrated by splitting it into leading-color and subleadingcolor pieces. The leading-color piece gives the numerically dominant contribution. The leading-color one-loop amplitudes are relatively simple and can therefore be integrated without further problems. The subleading-color one-loop amplitudes are more complicated and therefore need more computer time. On the other hand, they will yield contributions which are numerically smaller. We can thus evaluate them using fewer integrand evaluations. Although the second term $d \sigma^{R}-d \sigma^{A}$ usually gives only a modest numerical contribution, a naive Monte Carlo integration will give large statistical errors. Due to the large number of dipole factors involved, it is also very computationally intensive. We first deal with the fact that we have to reproduce logarithms numerically. This is solved by a remapping of phase-space. In a second stage we use color decomposition in order to reduce the number of dipole factors that have to be evaluated.

A simplified model for the term $d \sigma^{R}-d \sigma^{A}$ would be

$$
F=\int_{0}^{1} d x\left(\frac{f(x)}{x}-\frac{g(x)}{x}\right)
$$


where $f(0)=g(0)$ is assumed. The integral can be rewritten as

$$
F=\int_{0}^{y_{\min }} d x \frac{f(x)-g(x)}{x}+\int_{\ln y_{\min }}^{0} d y\left(f\left(e^{y}\right)-g\left(e^{y}\right)\right),
$$

where $y_{\text {min }}$ is an artificial parameter separating a numerically dangerous region from a stable region. Using the Taylor expansion for $f(x)-g(x)$, one sees that the first term gives a contribution of order $O\left(y_{\min }\right)$. In the second term the $1 / x$ behaviour has been absorbed into the integral measure by a change of variables $y=\ln x$, and the integrand tends to be more flat. This should reduce the statistical error in a Monte Carlo integration.

The precise remapping of phase space for the term $d \sigma^{R}-d \sigma^{A}$ is done as follows: Consider a set of products of invariants, including all the invariants in which the (unsubtracted) matrix elements has poles (and which may give rise to logarithms therefore). The relevant set of products of invariants for the two-quark, three-gluon final state consists of the pairs

$$
\begin{aligned}
\mathcal{S}_{g}= & \left\{s_{q 1} s_{12}, s_{q 1} s_{13}, s_{q 2} s_{21}, s_{q 2} s_{23}, s_{q 3} s_{31}, s_{q 3} s_{32}\right. \\
& s_{23} s_{3 \bar{q}}, s_{32} s_{2 \bar{q}}, s_{13} s_{3 \bar{q}}, s_{31} s_{1 \bar{q}}, s_{12} s_{2 \bar{q}}, s_{21} s_{1 \bar{q}} \\
& s_{12} s_{23}, s_{13} s_{32}, s_{21} s_{13} \\
& \left.s_{q 1} s_{1 \bar{q}}, s_{q 2} s_{2 \bar{q}}, s_{q 2} s_{2 \bar{q}}\right\}
\end{aligned}
$$

where we have labelled the gluons from 1 to $3, q$ corresponds to a quark and $\bar{q}$ to the anti-quark. Singularities associated with the last three pairs appear only in the colorsubleading part. The corresponding set for the four-quark, one-gluon final state consists of the pairs

$$
\begin{aligned}
\mathcal{S}_{q}= & \left\{s_{q g} s_{g \bar{q}}, s_{q^{\prime} g} s_{g \bar{q}^{\prime}}, s_{q g} s_{g \bar{q}^{\prime}}, s_{q^{\prime} g} s_{g \bar{q}}\right. \\
& s_{q \bar{q}} s_{\bar{q} q^{\prime}}, s_{q \bar{q}^{\prime}} s_{\bar{q}^{\prime} q^{\prime}}, s_{\bar{q} q} s_{q \bar{q}^{\prime}}, s_{\bar{q} q^{\prime}} s_{q^{\prime} \bar{q}^{\prime}} \\
& \left.s_{q g} s_{g q^{\prime}}, s_{\bar{q} g} s_{g \bar{q}^{\prime}}\right\} .
\end{aligned}
$$

The second line takes care of the collinear singularities when two quarks become collinear. The phase space is then partitioned according to

$$
\Phi_{n+1}=\sum_{\mathcal{S}} \Theta(a, b, c) \Phi_{n+1}
$$

where $\Theta(a, b, c)=1$ if $s_{a b} s_{b c}$ is the smallest product in the set $\mathcal{S}$, and $\Theta(a, b, c)=0$ otherwise. The sum is over all products in the set $\mathcal{S}$. We may use the symmetries under permutations in order to reduce the number of summands which need to be evaluated. For the two-quark, three-gluon case we have to evaluate the terms with $\Theta(q, 1,2)$ and $\Theta(2,3, \bar{q})$ with weight 6 , as well as the terms with $\Theta(1,2,3)$ and $\Theta(q, 3, \bar{q})$ which are weighted by a factor of 3 . For the four quark, one gluon case we evaluate the terms with $\Theta\left(q, g, \bar{q}^{\prime}\right), \Theta(q, g, \bar{q}), \Theta\left(q, \bar{q}, q^{\prime}\right)$ and $\Theta\left(\bar{q}, q, \bar{q}^{\prime}\right)$, which are weighted by a factor 2 , and the terms with $\Theta\left(q, g, q^{\prime}\right)$ and $\Theta\left(\bar{q}, g, \bar{q}^{\prime}\right)$ with unit weight.

Next, introduce a parameter $s_{\min }$ which separates numerically stable and unstable regions. (It is analogous to the $s_{\min }$ parameter in the phase-space slicing method, but 
plays a somewhat different role here.) Using this parameter, define two regions as follows. Suppose $s_{a s} s_{s b}$ is the smallest product in the set. Then the first region is defined as the region where $s_{a s}>s_{\min }$ and $s_{s b}>s_{\min }$. In this region we perform a remapping of the phase space as explained in detail in the next subsection.

The second region is the complement of the first: $s_{a s}<s_{\min }$ or $s_{s b}<s_{\min }$. In this region the integration is performed without any phase space remapping.

In summary, the numerical computation of the term $d \sigma^{R}-d \sigma^{A}$ goes as follows,

$$
\begin{aligned}
d \sigma^{R}-d \sigma^{A}= & \sum_{\mathcal{S}}\left(d \sigma^{R}-d \sigma^{A}\right) \Theta\left(s_{a s}-s_{\min }\right) \Theta\left(s_{s b}-s_{\min }\right) \Theta(a, s, b) \\
& +\sum_{\mathcal{S}}\left(d \sigma^{R}-d \sigma^{A}\right)\left(1-\Theta\left(s_{a s}-s_{\min }\right) \Theta\left(s_{s b}-s_{\min }\right)\right) \Theta(a, s, b) .
\end{aligned}
$$

Note that this separation into two regions does not involve any approximations and is exact whatever value $s_{\min }$ might take. The aim is of course to choose $s_{\min }$ so as to reduce the statistical errors. It turns out that choosing $s_{\min }$ small enough makes the contributions of the second region negligible. The contribution of the first region has a statistical error reduced by roughly a factor of ten compared to the result without any remapping and the same number of integrand evaluations. Empirically

$$
\eta=\frac{s_{\min } / Q^{2}}{y_{\text {cut }}}=10^{-5}
$$

is a good value, where $y_{\text {cut }}$ is the jet-defining parameter.

\subsection{Remapping of Phase Space}

The remapping of phase space we describe here was originally intended for use with the phase space slicing method, and was used in prior jet programs of one of the authors. The idea is to generate an $(n+1)$-parton configuration that is 'near' the hard $n$-parton configuration corresponding to the leading-order calculation. We want to do this in a way that makes the $(n+1)$-parton configuration approach the hard configuration in singular limits.

Suppose we have a set of product of invariants $\mathcal{S}$ such that $s_{a s} s_{s b}$ is the smallest product in the set. In this region we remap the phase space as follows. Let $k_{a}^{\prime}, k_{s}$ and $k_{b}^{\prime}$ be the corresponding momenta such that $s_{a s}=\left(k_{a}^{\prime}+k_{s}\right)^{2}, s_{s b}=\left(k_{b}^{\prime}+k_{s}\right)^{2}$ and $s_{a b}=\left(k_{a}^{\prime}+k_{s}+k_{b}^{\prime}\right)^{2}$. We want to relate this $(n+1)$ particle configuration to a nearby "hard" $n$-particle configuration with $\left(k_{a}+k_{b}\right)^{2}=\left(k_{a}^{\prime}+k_{s}+k_{b}^{\prime}\right)^{2}$, where $k_{a}$ and $k_{b}$ are the corresponding "hard" momenta. Using the factorization of the phase space, we have

$$
d \Phi_{n+1}=d \Phi_{n-1} \frac{d K^{2}}{2 \pi} d \Phi_{3}\left(K, k_{a}^{\prime}, k_{s}, k_{b}^{\prime}\right) .
$$

The three-particle phase space is given by

$$
\begin{aligned}
d \Phi_{3}\left(K, k_{a}^{\prime}, k_{s}, k_{b}^{\prime}\right) & =\frac{1}{32(2 \pi)^{5} s_{a b}} d s_{a s} d s_{s b} d \Omega_{b}^{\prime} d \phi_{s} \\
& =\frac{1}{4(2 \pi)^{3} s_{a b}} d s_{a s} d s_{s b} d \phi_{s} d \Phi_{2}\left(K, k_{a}, k_{b}\right)
\end{aligned}
$$


and therefore

$$
d \Phi_{n+1}=d \Phi_{n} \frac{d s_{a s} d s_{s b} d \phi_{s}}{4(2 \pi)^{3} s_{a b}} .
$$

The region of integration for $s_{a s}$ and $s_{s b}$ is $s_{a s}>s_{\min }, s_{s b}>s_{\min }$ (either from the $\Theta$ functions of phase space slicing method, or else from the $\Theta$-functions separating the two regions above) and $s_{a s}+s_{s b}<s_{a b}$ (Dalitz plot for massless particles). In order to get a flat integrand we want to absorb poles in $s_{a s}$ and $s_{s b}$ into the measure. This is done by changing the variables according to

$$
s_{a s}=s_{a b}\left(\frac{s_{\min }}{s_{a b}}\right)^{u_{1}}, \quad s_{s b}=s_{a b}\left(\frac{s_{\min }}{s_{a b}}\right)^{u_{2}}
$$

where $0 \leq u_{1}, u_{2} \leq 1$. Note that $u_{1}, u_{2}>0$ enforces $s_{a s}, s_{s b}>s_{\text {min. }}$. Therefore this transformation of variables may only be applied to invariants $s_{i j}$ where the region $0<$ $s_{i j}<s_{\min }$ is cut out. The phase space measure becomes

$$
d \Phi_{n+1}=d \Phi_{n} \frac{1}{4(2 \pi)^{3}} \frac{s_{a s} s_{s b}}{s_{a b}} \ln ^{2}\left(\frac{s_{\min }}{s_{a b}}\right) \Theta\left(s_{a s}+s_{s b}<s_{a b}\right) d u_{1} d u_{2} d \phi_{s} .
$$

This suggests the following algorithm for generating a $(n+1)$-parton configuration:

- Take a "hard" $n$-parton configuration and pick out two momenta $k_{a}$ and $k_{b}$. Use three uniformly distributed ('random') numbers $u_{1}, u_{2}, u_{3}$ and set

$$
\begin{aligned}
& s_{a b}=\left(k_{a}+k_{b}\right)^{2}, \\
& s_{a s}=s_{a b}\left(\frac{s_{\text {min }}}{s_{a b}}\right)^{u_{1}}, \\
& s_{s b}=s_{a b}\left(\frac{s_{\text {min }}}{s_{a b}}\right)^{u_{2}}, \\
& \phi_{s}=2 \pi u_{3} .
\end{aligned}
$$

- If $\left(s_{a s}+s_{s b}\right)>s_{a b}$, reject the event.

- If not, solve for $k_{a}^{\prime}, k_{b}^{\prime}$ and $k_{s}$. If $s_{a s}<s_{s b}$ we want to have $k_{b}^{\prime} \rightarrow k_{b}$ as $s_{a s} \rightarrow 0$. Define

$$
\begin{gathered}
E_{a}=\frac{s_{a b}-s_{s b}}{2 \sqrt{s_{a b}}}, \quad E_{s}=\frac{s_{a s}+s_{s b}}{2 \sqrt{s_{a b}}}, \quad E_{b}=\frac{s_{a b}-s_{a s}}{2 \sqrt{s_{a b}}}, \\
\theta_{a b}=\arccos \left(1-\frac{s_{a b}-s_{a s}-s_{s b}}{2 E_{a} E_{b}}\right), \quad \theta_{s b}=\arccos \left(1-\frac{s_{s b}}{2 E_{s} E_{b}}\right) .
\end{gathered}
$$

It is convenient to work in a coordinate system which is obtained by a Lorentz transformation to the center of mass of $k_{a}+k_{b}$ and a rotation such that $k_{b}^{\prime}$ is along the positive $z$-axis. In that coordinate system

$$
\begin{aligned}
p_{a}^{\prime} & =E_{a}\left(1, \sin \theta_{a b} \cos \left(\phi_{s}+\pi\right), \sin \theta_{a b} \sin \left(\phi_{s}+\pi\right), \cos \theta_{a b}\right), \\
p_{s} & =E_{s}\left(1, \sin \theta_{s b} \cos \phi_{s}, \sin \theta_{s b} \sin \phi_{s}, \cos \theta_{s b}\right), \\
p_{b}^{\prime} & =E_{b}(1,0,0,1) .
\end{aligned}
$$


The momenta $p_{a}^{\prime}, p_{s}$ and $p_{b}^{\prime}$ are related to the momenta $k_{a}^{\prime}, k_{s}$ and $k_{b}^{\prime}$ by a sequence of Lorentz transformations back to the original frame

$$
k_{a}^{\prime}=\Lambda_{\text {boost }} \Lambda_{x y}(\phi) \Lambda_{x z}(\theta) p_{a}^{\prime}
$$

and analogous for the other two momenta. The explicit formulæ for the Lorentz transformations are obtained as follows :

Let $K=\sqrt{\left(k_{a}+k_{b}\right)^{2}}$ and denote by $p_{b}$ the coordinates of the hard momentum $k_{b}$ in the center of mass system of $k_{a}+k_{b} . p_{b}$ is given by

$$
p_{b}=\left(\frac{Q^{0}}{K} k_{b}^{0}-\frac{\vec{k}_{b} \cdot \vec{Q}}{K}, \vec{k}_{b}+\left(\frac{\vec{k}_{b} \cdot \vec{Q}}{K\left(Q^{0}+K\right)}-\frac{k_{b}^{0}}{K}\right) \vec{Q}\right)
$$

with $Q=k_{a}+k_{b}$. The angles are then given by

$$
\begin{aligned}
\theta & =\arccos \left(1-\frac{p_{b} \cdot p_{b}^{\prime}}{2 p_{b}^{t} p_{b}^{t^{\prime}}}\right) \\
\phi & =\arctan \left(\frac{p_{b}^{y}}{p_{b}^{x}}\right)
\end{aligned}
$$

The explicit form of the rotations is

$$
\begin{aligned}
\Lambda_{x z}(\theta) & =\left(\begin{array}{cccc}
1 & 0 & 0 & 0 \\
0 & \cos \theta & 0 & \sin \theta \\
0 & 0 & 1 & 0 \\
0 & -\sin \theta & 0 & \cos \theta
\end{array}\right), \\
\Lambda_{x y}(\phi) & =\left(\begin{array}{cccc}
1 & 0 & 0 & 0 \\
0 & \cos \phi & -\sin \phi & 0 \\
0 & \sin \phi & \cos \phi & 0 \\
0 & 0 & 0 & 1
\end{array}\right) .
\end{aligned}
$$

The boost $k^{\prime}=\Lambda_{\text {boost }} q$ is given by

$$
k^{\prime}=\left(\frac{Q^{0}}{K} q^{0}+\frac{\vec{q} \cdot \vec{Q}}{K}, \vec{q}+\left(\frac{\vec{q} \cdot \vec{Q}}{K\left(Q^{0}+K\right)}+\frac{q^{0}}{K}\right) \vec{Q}\right)
$$

with $Q=k_{a}+k_{b}$ and $K=\sqrt{\left(k_{a}+k_{b}\right)^{2}}$.

- If $s_{a s}>s_{s b}$, exchange $a$ and $b$ in the formulæ above.

- The "soft" event has then the weight

$$
W_{n+1}=\frac{\pi}{2} \frac{1}{(2 \pi)^{3}} \frac{s_{a s} s_{s b}}{s_{a b}} \ln ^{2}\left(\frac{s_{\min }}{s_{a b}}\right) W_{n}
$$

where $W_{n}$ is the weight of the original "hard" event. 


\subsection{Color decomposition and soft or collinear limits}

We now turn our attention to the color decomposition of the five-parton tree amplitudes. Singling out the numerical dominant contribution, e.g. the leading color part, allows us to evaluate this part with fewer dipole factors as subtraction terms. This approach is similar to the one followed within the phase-space slicing method. In fact, we have also implemented phase-space slicing for the leading color contributions and checked that both methods, the dipole formalism and phase-space slicing, give the same numerical results. As a side-result we will give the relevant formulæ for the contributions from unresolved phase-space needed for the phase-space slicing method.

The color decomposition of the tree-level amplitude for $e^{+} e^{-} \rightarrow q g_{1} g_{2} g_{3} \bar{q}$ is

$$
\begin{aligned}
A_{5}\left(q, g_{1}, g_{2}, g_{3}, \bar{q}\right)= & \left(T^{1} T^{2} T^{3}\right)_{q \bar{q}} A_{5}^{(1)}(q, 1,2,3, \bar{q})+\left(T^{1} T^{3} T^{2}\right)_{q \bar{q}} A_{5}^{(2)}(q, 1,3,2, \bar{q}) \\
& +\left(T^{2} T^{3} T^{1}\right)_{q \bar{q}} A_{5}^{(3)}(q, 2,3,1, \bar{q})+\left(T^{2} T^{1} T^{3}\right)_{q \bar{q}} A_{5}^{(4)}(q, 2,1,3, \bar{q}) \\
& +\left(T^{3} T^{1} T^{2}\right)_{q \bar{q}} A_{5}^{(5)}(q, 3,1,2, \bar{q})+\left(T^{3} T^{2} T^{1}\right)_{q \bar{q}} A_{5}^{(6)}(q, 3,2,1, \bar{q}) .
\end{aligned}
$$

The index $i$ of $A_{5}^{(i)}$ labels the permutation of the gluons. Explicit formulæ for the partial amplitudes are given in ref. [10].

The tree-level amplitude for $e^{+} e^{-} \rightarrow g q \bar{q} q^{\prime} \bar{q}^{\prime}$ can be written as

$$
A_{5}\left(g, q, \bar{q}, q^{\prime}, \bar{q}^{\prime}\right)=\frac{1}{2} \delta_{14} T_{32} D_{1}+\frac{1}{2} \delta_{32} T_{14} D_{2}-\frac{1}{2} \delta_{12} T_{34} D_{3}-\frac{1}{2} \delta_{34} T_{12} D_{4}
$$

where the factor $1 / 2$ is due to the conventional normalization of $T^{a}$ and

$$
\begin{aligned}
D_{1}= & c_{0}(1) B_{1}(0 ; 1,2 ; 3,4)+c_{0}(3) B_{2}(0 ; 3,4 ; 1,2) \\
& +\delta_{\text {flav }} \frac{1}{N_{C}}\left(c_{0}(1) B_{3}(0 ; 1,4 ; 3,2)+c_{0}(3) B_{4}(0 ; 3,2 ; 1,4)\right), \\
D_{2}= & c_{0}(1) B_{2}(0 ; 1,2 ; 3,4)+c_{0}(3) B_{1}(0 ; 3,4 ; 1,2) \\
& +\delta_{\text {flav }} \frac{1}{N_{C}}\left(c_{0}(1) B_{4}(0 ; 1,4 ; 3,2)+c_{0}(3) B_{3}(0 ; 3,2 ; 1,4)\right), \\
D_{3}= & c_{0}(1) \frac{1}{N_{C}} B_{3}(0 ; 1,2 ; 3,4)+c_{0}(3) \frac{1}{N_{C}} B_{4}(0 ; 3,4 ; 1,2) \\
& +\delta_{\text {flav }}\left(c_{0}(1) B_{1}(0 ; 1,4 ; 3,2)+c_{0}(3) B_{2}(0 ; 3,2 ; 1,4)\right), \\
D_{4}= & c_{0}(1) \frac{1}{N_{C}} B_{4}(0 ; 1,2 ; 3,4)+c_{0}(3) \frac{1}{N_{C}} B_{3}(0 ; 3,4 ; 1,2) \\
& +\delta_{\text {flav }}\left(c_{0}(1) B_{2}(0 ; 1,4 ; 3,2)+c_{0}(3) B_{1}(0 ; 3,2 ; 1,4)\right) .
\end{aligned}
$$

Explicit formulæ for the partial amplitudes $B_{i}$ are again given in ref. [10]. In this equation, $c_{0}(j)$ denotes a factor from the electro-weak coupling and depends explicitly on the flavor of the quark $q_{j}$,

$$
\begin{aligned}
c_{0}(j) & =-Q^{q_{j}}+v^{e} v^{q_{j}} \mathcal{P}_{Z}\left(s_{56}\right) \\
\mathcal{P}_{Z}(s) & =\frac{s}{s-M_{Z}^{2}+i \Gamma_{Z} M_{Z}} .
\end{aligned}
$$

The electron - positron pair can either annihilate into a photon or a $Z$-boson. The first term in the expression for $c_{0}$ corresponds to an intermediate photon, whereas the last 
term corresponds to a $Z$-boson. The left- and right-handed couplings of the $Z$-boson to fermions are given by

$$
v_{L}=\frac{I_{3}-Q \sin ^{2} \theta_{W}}{\sin \theta_{W} \cos \theta_{W}}, \quad v_{R}=\frac{-Q \sin \theta_{W}}{\cos \theta_{W}}
$$

where $Q$ and $I_{3}$ are the charge and the third component of the weak isospin of the fermion. For an electron and up- or down-type quarks we have:

$$
\begin{array}{ccc} 
& Q & I_{3} \\
\left(\begin{array}{c}
u \\
d
\end{array}\right) & \left(\begin{array}{c}
2 / 3 \\
-1 / 3
\end{array}\right) & \left(\begin{array}{c}
1 / 2 \\
-1 / 2
\end{array}\right) \\
e^{-} & -1 & -1 / 2
\end{array}
$$

Next, consider the soft and collinear limit of any of these partial amplitudes, e.g. $A_{5}^{(i)}$ or $B_{i}$. In the soft-gluon limit, a partial amplitude factorizes,

$$
A_{n+1} \rightarrow g \cdot \operatorname{Eik}_{a b}^{\lambda} \cdot A_{n},
$$

where the eikonal factors are

$$
\operatorname{Eik}_{a b}^{+}=\sqrt{2} \frac{\langle a b\rangle}{\langle a s\rangle\langle s b\rangle}, \quad \operatorname{Eik}_{a b}^{-}=-\sqrt{2} \frac{[a b]}{[a s][s b]} .
$$

Squaring the amplitude we obtain terms like

$$
\begin{aligned}
& \sum_{\text {soft gluon helicity } \lambda} A_{n+1}^{(2) *} A_{n+1}^{(1)}+A_{n+1}^{(1) *} A_{n+1}^{(2)} \\
& =g^{2}\left(\operatorname{Eik}^{(2)-} \operatorname{Eik}^{(1)+}+\operatorname{Eik}^{(2)+} \operatorname{Eik}^{(1)-}\right)\left(A_{n}^{(2) *} A_{n}^{(1)}+A_{n}^{(1) *} A_{n}^{(2)}\right) .
\end{aligned}
$$

There are three different cases, depending how the soft gluon is inserted into the partial amplitude $A_{n+1}^{(1)}$ and $A_{n+1}^{(2)}$.

1. Two common legs, e.g. $A_{n+1}^{(1)}=A_{n+1}^{(1)}(\ldots, a, s, b, \ldots)$ and $A_{n+1}^{(2)}=A_{n+1}^{(2)}(\ldots, a, s, b, \ldots)$,

$$
\sum_{\lambda} A_{n+1}^{(2) *} A_{n+1}^{(1)}+A_{n+1}^{(1) *} A_{n+1}^{(2)}=g^{2} \cdot 2 \cdot 2 \frac{s_{a b}}{s_{a s} s_{s b}}\left(A_{n}^{(2) *} A_{n}^{(1)}+A_{n}^{(1) *} A_{n}^{(2)}\right)
$$

2. One common leg, e.g. $A_{n+1}^{(1)}=A_{n+1}^{(1)}(\ldots, a, s, b, \ldots)$ and $A_{n+1}^{(2)}=A_{n+1}^{(2)}(\ldots, a, s, c, \ldots)$,

$$
\begin{aligned}
& \sum_{\lambda} A_{n+1}^{(2) *} A_{n+1}^{(1)}+A_{n+1}^{(1) *} A_{n+1}^{(2)}= \\
& \quad=g^{2} \cdot 2\left(\frac{\langle a b\rangle}{\langle a s\rangle\langle s b\rangle} \frac{[c a]}{[c s][s a]}+\frac{[a b]}{[a s][s b]} \frac{\langle c a\rangle}{\langle c s\rangle\langle s a\rangle}\right)\left(A_{n}^{(2) *} A_{n}^{(1)}+A_{n}^{(1) *} A_{n}^{(2)}\right) \\
& \quad=g^{2} \cdot 2\left(\frac{s_{a b}}{s_{a s} s_{s b}}-\frac{s_{b c}}{s_{b s} s_{s c}}+\frac{s_{a c}}{s_{a s} s_{s c}}\right)\left(A_{n}^{(2) *} A_{n}^{(1)}+A_{n}^{(1) *} A_{n}^{(2)}\right)
\end{aligned}
$$


3. No common legs, e.g. $A_{n+1}^{(1)}=A_{n+1}^{(1)}(\ldots, a, s, b, \ldots)$ and $A_{n+1}^{(2)}=A_{n+1}^{(2)}(\ldots, d, s, c, \ldots)$,

$$
\begin{aligned}
& \sum_{\lambda} A_{n+1}^{(2) *} A_{n+1}^{(1)}+A_{n+1}^{(1) *} A_{n+1}^{(2)}= \\
& =g^{2} \cdot 2\left(\frac{\langle a b\rangle}{\langle a s\rangle\langle s b\rangle} \frac{[c d]}{[c s][s d]}+\frac{[a b]}{[a s][s b]} \frac{\langle c d\rangle}{\langle c s\rangle\langle s d\rangle}\right)\left(A_{n}^{(2) *} A_{n}^{(1)}+A_{n}^{(1) *} A_{n}^{(2)}\right) \\
& =g^{2} \cdot 2\left(\frac{s_{a c}}{s_{a s} s_{s c}}-\frac{s_{a d}}{s_{a s} s_{s d}}+\frac{s_{b d}}{s_{b s} s_{s d}}-\frac{s_{b c}}{s_{b s} s_{s c}}\right)\left(A_{n}^{(2) *} A_{n}^{(1)}+A_{n}^{(1) *} A_{n}^{(2)}\right)
\end{aligned}
$$

In the collinear limit tree-level partial amplitudes factorize like

$$
A_{n+1} \rightarrow g \sum_{\lambda=+/-} \operatorname{Split}_{-\lambda}\left(p_{a}^{\lambda_{a}}, p_{b}^{\lambda_{b}}\right) A_{n}\left(\ldots, P^{\lambda}, \ldots\right)
$$

where $P=p_{a}+p_{b}, p_{a}=z P$ and $p_{b}=(1-z) P . \lambda, \lambda_{a}$ and $\lambda_{b}$ denote the corresponding helicities. The splitting functions are [11]:

$$
\begin{aligned}
\operatorname{Split}_{g^{+}}\left(g^{+}, g^{+}\right)=0 & \operatorname{Split}_{g^{-}}\left(g^{-}, g^{-}\right)=0 \\
\operatorname{Split}_{g^{+}}\left(g^{+}, g^{-}\right)=\sqrt{2} \frac{(1-z)^{\frac{3}{2}}}{\sqrt{z}\langle a b\rangle} & \operatorname{Split}_{g^{-}}\left(g^{-}, g^{+}\right)=-\sqrt{2} \frac{(1-z)^{\frac{3}{2}}}{\sqrt{z}[a b]} \\
\operatorname{Split}_{g^{+}}\left(g^{-}, g^{+}\right)=\sqrt{2} \frac{z^{\frac{3}{2}}}{\sqrt{(1-z)}\langle a b\rangle} & \operatorname{Split}_{g^{-}}\left(g^{+}, g^{-}\right)=-\sqrt{2} \frac{z^{\frac{3}{2}}}{\sqrt{(1-z)}[a b]} \\
\operatorname{Split}_{g^{+}}\left(g^{-}, g^{-}\right)=-\sqrt{2} \frac{1}{\sqrt{z(1-z)}[a b]} & \operatorname{Split}_{g^{-}}\left(g^{+}, g^{+}\right)=\sqrt{2} \frac{1}{\sqrt{z(1-z)}\langle a b\rangle} \\
\operatorname{Split}_{q^{-}}\left(q^{+}, g^{+}\right)=\sqrt{2} \frac{1}{\sqrt{1-z}\langle a b\rangle} & \operatorname{Split}_{q^{+}}\left(q^{-}, g^{-}\right)=-\sqrt{2} \frac{1}{\sqrt{1-z}[a b]} \\
\operatorname{Split}_{q^{-}}\left(q^{+}, g^{-}\right)=-\sqrt{2} \frac{z}{\sqrt{1-z}[a b]} & \operatorname{Split}_{q^{+}}\left(q^{-}, g^{+}\right)=\sqrt{2} \frac{z}{\sqrt{1-z}\langle a b\rangle} \\
\operatorname{Split}_{\bar{q}^{-}}\left(g^{+}, \bar{q}^{+}\right)=\sqrt{2} \frac{1}{\sqrt{z}\langle a b\rangle} & \operatorname{Split}_{\bar{q}^{+}}\left(g^{-}, \bar{q}^{-}\right)=-\sqrt{2} \frac{1}{\sqrt{z}[a b]} \\
\operatorname{Split}_{\bar{q}^{-}}\left(g^{-}, \bar{q}^{+}\right)=-\sqrt{2} \frac{1-z}{\sqrt{z}[a b]} & \operatorname{Split}_{\bar{q}^{+}}\left(g^{+}, \bar{q}^{-}\right)=\sqrt{2} \frac{1-z}{\sqrt{z}\langle a b\rangle} \\
\operatorname{Split}_{g^{+}}\left(q^{+}, \bar{q}^{-}\right)=\sqrt{2} \frac{1-z}{\langle a b\rangle} & \operatorname{Split}_{g^{-}}\left(q^{-}, \bar{q}^{+}\right)=-\sqrt{2} \frac{1-z}{[a b]} \\
\operatorname{Split}_{g^{+}}\left(q^{-}, \bar{q}^{+}\right)=-\sqrt{2} \frac{z}{\langle a b\rangle} & \operatorname{Split}_{g^{-}}\left(q^{+}, \bar{q}^{-}\right)=\sqrt{2} \frac{z}{[a b]}
\end{aligned}
$$

We have checked numerically that all five-parton tree amplitudes in the program have the correct collinear limits.

\subsection{Real Emission with Two Quarks and Three Gluons}

The matrix element squared of the tree-level amplitude for $e^{+} e^{-} \rightarrow q g_{1} g_{2} g_{3} \bar{q}$ can be written as

$$
\left|A_{5}\left(q, g_{1}, g_{2}, g_{3}, \bar{q}\right)\right|^{2}=\vec{A}_{5}^{\dagger} C \vec{A}_{5}
$$


where

$$
\overrightarrow{A_{5}}=\left(\begin{array}{c}
A_{5}^{(1)}(q, 1,2,3, \bar{q}) \\
A_{5}^{(2)}(q, 1,3,2, \bar{q}) \\
A_{5}^{(3)}(q, 2,3,1, \bar{q}) \\
A_{5}^{(4)}(q, 2,1,3, \bar{q}) \\
A_{5}^{(5)}(q, 3,1,2, \bar{q}) \\
A_{5}^{(6)}(q, 3,2,1, \bar{q})
\end{array}\right)
$$

and the color matrix is given by

$$
C=\left(\begin{array}{cccccc}
c_{1}^{(3)} & c_{2}^{(3)} & c_{3}^{(3)} & c_{2}^{(3)} & c_{3}^{(3)} & c_{4}^{(3)} \\
c_{2}^{(3)} & c_{1}^{(3)} & c_{4}^{(3)} & c_{3}^{(3)} & c_{2}^{(3)} & c_{3}^{(3)} \\
c_{3}^{(3)} & c_{4}^{(3)} & c_{1}^{(3)} & c_{2}^{(3)} & c_{3}^{(3)} & c_{2}^{(3)} \\
c_{2}^{(3)} & c_{3}^{(3)} & c_{2}^{(3)} & c_{1}^{(3)} & c_{4}^{(3)} & c_{3}^{(3)} \\
c_{3}^{(3)} & c_{2}^{(3)} & c_{3}^{(3)} & c_{4}^{(3)} & c_{1}^{(3)} & c_{2}^{(3)} \\
c_{4}^{(3)} & c_{3}^{(3)} & c_{2}^{(3)} & c_{3}^{(3)} & c_{2}^{(3)} & c_{1}^{(3)}
\end{array}\right)
$$

where the color factors are

$$
\begin{aligned}
c_{1}^{(3)} & =\left(\frac{1}{2}\right)^{3} \frac{\left(N^{2}-1\right)^{3}}{N^{2}}, \\
c_{2}^{(3)} & =-\left(\frac{1}{2}\right)^{3} \frac{\left(N^{2}-1\right)^{2}}{N^{2}}, \\
c_{3}^{(3)} & =\left(\frac{1}{2}\right)^{3} \frac{N^{2}-1}{N^{2}} \\
c_{4}^{(3)} & =\left(\frac{1}{2}\right)^{3} \frac{\left(N^{2}-1\right)\left(N^{2}+1\right)}{N^{2}}=2 c_{3}^{(3)}-c_{2}^{(3)} .
\end{aligned}
$$

If one gluon becomes soft or collinear, the matrix element squared reduces to the one with one gluon less

$$
\left|A_{5}\right|^{2} \rightarrow \vec{A}_{4}^{\dagger}\left(\begin{array}{cc}
c_{1}^{(2)} R_{\text {diag }}(q, 1,2, \bar{q}) & c_{2}^{(2)} R_{o f f}(q, 1,2, \bar{q}) \\
c_{2}^{(2)} R_{o f f}(q, 1,2, \bar{q}) & c_{1}^{(2)} R_{\text {diag }}(q, 2,1, \bar{q})
\end{array}\right) \vec{A}_{4} .
$$

where the color factors are

$$
\begin{aligned}
c_{1}^{(2)} & =\left(\frac{1}{2}\right)^{2} \frac{\left(N^{2}-1\right)^{2}}{N}, \\
c_{2}^{(2)} & =-\left(\frac{1}{2}\right)^{2} \frac{N^{2}-1}{N}
\end{aligned}
$$

The finite $R$-factors, which give the contribution from unresolved phase-space within the phase-space slicing method, are given by

$$
\begin{aligned}
& R_{\text {diag }}(q, 1,2, \bar{q})= \\
& \quad=\frac{g^{2} c_{\Gamma}}{c_{1}^{(2)}}\left\{2 c_{1}(3) R_{2}(q, 1,2, \bar{q})\right.
\end{aligned}
$$




$$
\begin{aligned}
& -2 c_{2}^{(3)}\left(2 R_{2}(q, 1,2, \bar{q})-R_{1}(q, 1, \bar{q})-R_{1}(q, 2, \bar{q})\right) \\
& +2 c_{3}^{(3)}\left(R_{2}(q, 1,2, \bar{q})-R_{1}(q, 1, \bar{q})-R_{1}(q, 2, \bar{q})+R_{0}(q, \bar{q})\right) \\
& \left.+2 I_{g \rightarrow q \bar{q}}\right\} \\
R_{o f f}( & (q, 1,2, \bar{q})= \\
= & \frac{g^{2} c_{\Gamma}}{c_{2}^{(2)}}\left\{-c_{2}^{(3)}\left(R_{2}(q, 1,2, \bar{q})+R_{2}(q, 2,1, \bar{q})-2 R_{1}(q, 1, \bar{q})-2 R_{1}(q, 2, \bar{q})\right)\right. \\
& +2 c_{3}^{(3)}\left(R_{2}(q, 1,2, \bar{q})+R_{2}(q, 2,1, \bar{q})-R_{1}(q, 1, \bar{q})-R_{1}(q, 2, \bar{q})\right) \\
& -c_{4}^{(3)}\left(R_{2}(q, 1,2, \bar{q})+R_{2}(q, 2,1, \bar{q})-2 R_{0}(q, \bar{q})\right) \\
& \left.+2 I_{g \rightarrow q \bar{q}}\right\} .
\end{aligned}
$$

We have written the contribution from each color factor separately. The last line corresponds to the contribution from the $A\left(g, q, \bar{q}, q^{\prime}, \bar{q}^{\prime}\right)$ amplitudes, when one pair of quarks become collinear. We have used the notation

$$
\begin{aligned}
R_{0}(q, \bar{q})= & S(q, \bar{q})-\frac{4}{N_{c}} \frac{C_{A}}{2 C_{F}} 2 I_{q \rightarrow q g}\left(\frac{s_{\min }}{s_{q \bar{q}}}\right), \\
R_{1}(q, 1, \bar{q})= & S(q, 1)+S(1, \bar{q})-\frac{4}{N_{c}}\left[\frac{C_{A}}{2 C_{F}} I_{q \rightarrow q g}\left(\frac{s_{\min }}{s_{q 1}}\right)\right. \\
& \left.+I_{g \rightarrow g g}\left(\frac{s_{\min }}{s_{q 1}}, \frac{s_{\min }}{s_{1 \bar{q}}}\right)+\frac{C_{A}}{2 C_{F}} I_{q \rightarrow q g}\left(\frac{s_{\min }}{s_{1 \bar{q}}}\right)\right], \\
R_{2}(q, 1,2, \bar{q})= & S(q, 1)+S(1,2)+S(2, \bar{q})-\frac{4}{N_{c}}\left[\frac{C_{A}}{2 C_{F}} I_{q \rightarrow q g}\left(\frac{s_{\min }}{s_{q 1}}\right)\right. \\
& +I_{g \rightarrow g g}\left(\frac{s_{\min }}{s_{q 1}}, \frac{s_{\min }}{s_{12}}\right)+I_{g \rightarrow g g}\left(\frac{s_{\min }}{s_{12}}, \frac{s_{\min }}{s_{2 \bar{q}}}\right) \\
& \left.+\frac{C_{A}}{2 C_{F}} I_{q \rightarrow q g}\left(\frac{s_{\min }}{s_{2 \bar{q}}}\right)\right], \\
S(a, b)= & \ln ^{2}\left(\frac{\mu^{2}}{s_{\min }}\right)+\ln ^{2}\left(\frac{s_{a b}}{s_{\min }}\right)+2 \ln \left(\frac{\mu^{2}}{s_{\min }}\right) \ln \left(\frac{s_{a b}}{s_{\min }}\right), \\
I_{a \rightarrow b c}\left(z_{1}, z_{2}\right)= & I_{a \rightarrow b c}^{1}\left(z_{1}, z_{2}\right)+I_{a \rightarrow b c}^{0}\left(z_{1}, z_{2}\right) \ln \left(\frac{\mu^{2}}{s_{\min }}\right) .
\end{aligned}
$$

$I_{a \rightarrow b c}^{0}$ and $I_{a \rightarrow b c}^{1}$ are the terms of order $O\left(\varepsilon^{0}\right)$ and $O\left(\varepsilon^{1}\right)$ obtained from the $D$-dimensional splitting functions integrated over unresolved phase space. In the conventional scheme they are given by

$$
\begin{aligned}
& I_{g \rightarrow g g}=\frac{C_{A}}{2}\left(-\ln z_{1}-\ln z_{2}-\frac{11}{6}+\left(\frac{1}{2} \ln ^{2} z_{1}+\frac{1}{2} \ln ^{2} z_{2}-\frac{67}{18}+\frac{\pi^{2}}{3}\right) \varepsilon+O\left(\varepsilon^{2}\right)\right), \\
& I_{q \rightarrow q g}=C_{F}\left(-\ln z_{2}-\frac{3}{4}+\left(\frac{1}{2} \ln ^{2} z_{2}-\frac{7}{4}+\frac{\pi^{2}}{6}\right) \varepsilon+O\left(\varepsilon^{2}\right)\right) \\
& I_{g \rightarrow q \bar{q}}=T_{R} N_{f}\left(\frac{1}{3}+\frac{5}{9} \varepsilon+O\left(\varepsilon^{2}\right)\right) .
\end{aligned}
$$




\subsection{Real Emission with Four Quarks and One Gluon}

The matrix element squared of the tree-level amplitude for $e^{+} e^{-} \rightarrow g q \bar{q} q^{\prime} \bar{q}^{\prime}$ can be written as

$$
\left|A_{5}\left(g, q, \bar{q}, q^{\prime}, \bar{q}^{\prime}\right)\right|^{2}=\vec{D}^{\dagger} C \vec{D}
$$

where

$$
\vec{D}=\left(\begin{array}{c}
D_{1} \\
D_{2} \\
D_{3} \\
D_{4}
\end{array}\right)
$$

Then the color matrix is given by

$$
C=\left(\begin{array}{cccc}
c_{1}^{(1)} & 0 & c_{2}^{(1)} & c_{2}^{(1)} \\
0 & c_{1}^{(1)} & c_{2}^{(1)} & c_{2}^{(1)} \\
c_{2}^{(1)} & c_{2}^{(1)} & c_{1}^{(1)} & 0 \\
c_{2}^{(1)} & c_{2}^{(1)} & 0 & c_{1}^{(1)}
\end{array}\right)
$$

where

$$
c_{1}^{(1)}=\frac{1}{8} N_{C}\left(N_{C}^{2}-1\right), \quad c_{2}^{(1)}=-\frac{1}{8}\left(N_{C}^{2}-1\right)
$$

The color decomposition of the four quark amplitude can be written as

$$
\begin{aligned}
A_{4}= & \frac{1}{2}\left(\delta_{12} \delta_{34}-\frac{1}{N_{c}} \delta_{14} \delta_{32}\right) \chi(1,2,3,4) \\
& -\frac{1}{2}\left(\delta_{14} \delta_{32}-\frac{1}{N_{c}} \delta_{12} \delta_{34}\right) \delta_{\text {flav }} \chi(1,4,3,2)
\end{aligned}
$$

where

$$
\chi(1,2,3,4)=A_{4}(1,2,3,4)+A_{4}(3,4,1,2) .
$$

The matrix element squared we may write as

$$
\left|A_{4}\right|^{2}=\vec{\chi}^{\dagger}\left(\begin{array}{cc}
c_{1}^{(0)} & c_{2}^{(0)} \\
c_{2}^{(0)} & c_{1}^{(0)}
\end{array}\right) \vec{\chi}
$$

with

$$
c_{1}^{(0)}=\frac{1}{4}\left(N_{c}^{2}-1\right), \quad c_{2}^{(0)}=\frac{1}{4} \frac{N_{c}^{2}-1}{N_{c}}
$$

and

$$
\vec{\chi}=\left(\begin{array}{c}
\chi(1,2,3,4) \\
\delta_{\text {flav }} \chi(1,4,3,2)
\end{array}\right)
$$


In the soft gluon limit, the functions $B_{i}$ behave like

$$
\begin{aligned}
& B_{1}(0 ; 1,2 ; 3,4) \rightarrow g \cdot \operatorname{Eik}_{32} \cdot A(1,2 ; 3,4), \\
& B_{2}(0 ; 1,2 ; 3,4) \rightarrow g \cdot \operatorname{Eik}_{14} \cdot A(1,2 ; 3,4), \\
& B_{3}(0 ; 1,2 ; 3,4) \rightarrow g \cdot \operatorname{Eik}_{34} \cdot A(1,2 ; 3,4), \\
& B_{4}(0 ; 1,2 ; 3,4) \rightarrow g \cdot \operatorname{Eik}_{12} \cdot A(1,2 ; 3,4) .
\end{aligned}
$$

In the limit where one pair of quarks becomes collinear, the amplitudes factorize as

$$
\begin{aligned}
& B_{1}(0 ; 1,2 ; 3,4) \rightarrow g \sum_{\lambda} \operatorname{Split}_{P}(3,4) A_{4}(1, P, 0,2) \\
& B_{2}(0 ; 1,2 ; 3,4) \rightarrow g \sum_{\lambda} \operatorname{Split}_{P}(3,4) A_{4}(1,0, P, 2) \\
& B_{3}(0 ; 1,2 ; 3,4) \rightarrow 0, \\
& B_{4}(0 ; 1,2 ; 3,4) \rightarrow g \sum_{\lambda} \operatorname{Split}_{P}(3,4)\left(A_{4}(1, P, 0,2)+A_{4}(1,0, P, 2)\right) .
\end{aligned}
$$

One of the functions $B_{i}$ is redundant, since

$$
B_{1}+B_{2}-B_{3}-B_{4}=0
$$

The contribution from unresolved phase space is written as

$$
\vec{\chi}^{\dagger}\left(\begin{array}{cc}
c_{1}^{(0)} R_{\text {diag }}(1,2,3,4) & c_{2}^{(0)} R_{o f f}(1,2,3,4) \\
c_{2}^{(0)} R_{o f f}(1,2,3,4) & c_{1}^{(0)} R_{\text {diag }}(1,4,3,2)
\end{array}\right) \vec{\chi}
$$

with

$$
\begin{aligned}
& R_{\text {diag }}(1,2,3,4)= \\
& =\frac{g^{2} c_{\Gamma}}{c_{1}^{(0)}}\left\{2 c_{1}^{(1)}\left[R_{0}(1,4)+R_{0}(2,3)+\frac{1}{N_{c}^{2}}\left(R_{0}(1,2)+R_{0}(3,4)\right)\right]\right. \\
& \left.\quad+4 \frac{c_{2}^{(1)}}{N_{c}}\left[R_{0}(1,4)+R_{0}(2,3)+R_{0}(1,2)+R_{0}(3,4)-R_{0}(1,3)-R_{0}(2,4)\right]\right\}, \\
& R_{o f f}(1,2,3,4)= \\
& =\frac{g^{2} c_{\Gamma}}{c_{1}^{(0)}}\left\{2 \frac{c_{1}^{(1)}}{N_{c}}\left[R_{0}(1,4)+R_{0}(2,3)+R_{0}(1,2)+R_{0}(3,4)\right]\right. \\
& \left.\quad+2 c_{2}^{(2)}\left(1+\frac{1}{N_{c}^{2}}\right)\left[R_{0}(1,4)+R_{0}(2,3)+R_{0}(1,2)+R_{0}(3,4)-R_{0}(1,3)-R_{0}(2,4)\right]\right\} .
\end{aligned}
$$

\subsection{Color Correlation}

The color correlation matrices may be obtained by two ways. The first approach is the one given by Catani and Seymour. Within this approach one acts with the color charge 
operators on the $n$-parton amplitudes. For example

$$
\begin{gathered}
\left\langle q, 1,2, \bar{q}\left|T_{q} \cdot T_{1}\right| q, 1,2, \bar{q}\right\rangle=\left(T_{\bar{q} j}^{2} T_{j q^{\prime}}^{1^{\prime}} A(q, 1,2, \bar{q})^{*}, T_{\bar{q} j}^{1^{\prime}} T_{j q^{\prime}}^{2} A(q, 2,1, \bar{q})^{*}\right) \\
\cdot T_{q^{\prime} q}^{a} \cdot 2 \operatorname{Tr}\left(T^{1^{\prime}} T^{a} T^{1}-T^{a} T^{1^{\prime}} T^{1}\right) \cdot\left(\begin{array}{c}
T_{q i}^{1} T_{i \bar{q}}^{2} A(q, 1,2, \bar{q}) \\
T_{q i}^{2} T_{i \bar{q}}^{1} A(q, 2,1, \bar{q})
\end{array}\right)
\end{gathered}
$$

Here the color charge operator for the gluon has been written as

$$
i f^{c a b}=2 \operatorname{Tr}\left(T^{c} T^{a} T^{b}-T^{a} T^{c} T^{b}\right)
$$

On the other hand one may start from the color decomposition of the $(n+1)$-parton matrix element in the form of equations (46) and (60). One then considers the soft and collinear limits using the partial fraction decompositions (41) - (43). This procedure is identical to the one followed in the phase space slicing approach. With the help of the identity

$$
\frac{s_{a b}}{s_{a s} s_{s b}}=\frac{s_{a b}}{s_{a s}\left(s_{a s}+s_{s b}\right)}+\frac{s_{a b}}{s_{s b}\left(s_{a s}+s_{s b}\right)}
$$

the color correlation matrix can then be read off from equations (50) and (72). This approach has the advantage that it makes the connection between each divergent term in the $(n+1)$-parton matrix element and the corresponding subtraction term transparent.

The leading order matrix element $e^{+} e^{-} \rightarrow q g_{1} g_{2} g_{3} \bar{q}$ needs 27 dipole factors. There are six terms where the quark is the emitter and a gluon the spectator and three terms where the role of the emitter and spectator are exchanged. The color correlation matrices are invariant under the exchange of emitter and spectator. The color correlation matrix for the case $T_{q} \cdot T_{1}$ is given by

$$
T_{q} \cdot T_{1}=\left(\begin{array}{cc}
c_{2}^{(3)}-c_{1}^{(3)} & \frac{1}{2}\left(c_{4}^{(3)}-c_{2}^{(3)}\right) \\
\frac{1}{2}\left(c_{4}^{(3)}-c_{2}^{(3)}\right) & c_{3}^{(3)}-c_{2}^{(3)}
\end{array}\right)
$$

If the antiquark $\bar{q}$ is the spectator we obtain

$$
T_{q} \cdot T_{\bar{q}}=\left(\begin{array}{cc}
-c_{3}^{(3)} & -c_{4}^{(3)} \\
-c_{4}^{(3)} & -c_{3}^{(3)}
\end{array}\right)
$$

Finally the color correlation matrix where both emitter and spectator are gluons is given by

$$
T_{1} \cdot T_{2}=\left(\begin{array}{cc}
-c_{1}^{(3)}+2 c_{2}^{(3)}-c_{3}^{(3)} & 0 \\
0 & -c_{1}^{(3)}+2 c_{2}^{(3)}-c_{3}^{(3)}
\end{array}\right)
$$

All other color correlation matrices can be obtained by a permutation of indices.

The leading order matrix element $e^{+} e^{-} \rightarrow g q \bar{q} q^{\prime} \bar{q}^{\prime}$ needs 12 dipole factors associated with the splitting $g \rightarrow q \bar{q}$ and 12 dipole factors associated to the splitting $q \rightarrow q g$ or 
$\bar{q} \rightarrow g \bar{q}$. The relevant color correlation matrices for the first case where already given above. The color correlation matrices for the latter case are

$$
\begin{gathered}
T_{q} \cdot T_{\bar{q}}=\left(\begin{array}{cc}
-\frac{c_{1}^{(1)}}{N_{c}^{2}}-2 \frac{c_{2}^{(1)}}{N_{c}} & -\frac{c_{1}^{(1)}}{N_{c}}-c_{2}^{(1)}\left(1+\frac{1}{N_{c}^{2}}\right) \\
-\frac{c_{1}^{(1)}}{N_{c}}-c_{2}^{(1)}\left(1+\frac{1}{N_{c}^{2}}\right) & -c_{1}^{(1)}-2 \frac{c_{2}^{(1)}}{N_{c}}
\end{array}\right) \\
T_{q} \cdot T_{\bar{q}^{\prime}}=\left(\begin{array}{cc}
-c_{1}^{(1)}-2 \frac{c_{2}^{(1)}}{N_{c}} & -\frac{c_{1}^{(1)}}{N_{c}}-c_{2}^{(1)}\left(1+\frac{1}{N_{c}^{2}}\right) \\
-\frac{c_{1}^{(1)}}{N_{c}}-c_{2}^{(1)}\left(1+\frac{1}{N_{c}^{2}}\right) & -\frac{c_{1}^{(1)}}{N_{c}^{2}}-2 \frac{c_{2}^{(1)}}{N_{c}}
\end{array}\right) \\
T_{q} \cdot T_{q^{\prime}}=\left(\begin{array}{cc}
2 \frac{c_{2}^{(1)}}{N_{c}} & c_{2}^{(1)}\left(1+\frac{1}{N_{c}^{2}}\right) \\
c_{2}^{(1)}\left(1+\frac{1}{N_{c}^{2}}\right) & 2 \frac{c_{2}^{(1)}}{N_{c}}
\end{array}\right)
\end{gathered}
$$

We can now pick out partial amplitudes corresponding to one specific color factor. This subset will in general require fewer dipole factors as subtraction terms. In practise it is enough to single out the leading color contribution for the matrix element $e^{+} e^{-} \rightarrow$ $q g_{1} g_{2} g_{3} \bar{q}$. Using the symmetry under the permutation of the gluons it is sufficient to evaluate one partial amplitude, which we may take to be

$$
A_{5}^{(1)}(q, 1,2,3, \bar{q})
$$

weighted by a factor of six. This partial amplitude needs only six dipole factors $V_{i j, k}$, where $(i j)$ denotes the emitter and $k$ the spectator:

$$
V_{q 1,2}, V_{12, q}, V_{12,3}, V_{23,1}, V_{23, \bar{q}}, V_{3 \bar{q}, 2}
$$

The color correlation matrices simplify accordingly, for example

$$
T_{q} \cdot T_{1}=\left(\begin{array}{cc}
-c_{1}^{(3)} & 0 \\
0 & 0
\end{array}\right)
$$

which requires only the evaluation of one partial amplitude of the four-parton tree-level amplitude. We may further restrict the set of products of invariants to

$$
\mathcal{S}_{g}=\left\{s_{q 1} s_{12}, s_{12} s_{23}, s_{23} s_{3 \bar{q}}\right\},
$$

since we can only have poles in $s_{q 1}, s_{12}, s_{23}$ or $s_{3 \bar{q}}$. Singling out the leading color contributions for $e^{+} e^{-} \rightarrow q g_{1} g_{2} g_{3} \bar{q}$ together with the remapping of phase space discussed in section 2.3 improves the efficiency of the Monte Carlo integration of the term $d \sigma^{R}-d \sigma^{A}$ sufficiently so that most of the computer time is spent on the evaluation of the virtual one-loop amplitudes.

We finally remark that Z. Nagy and Z. Trócsányi [4] have chosen a different approach in order to improve the efficiency of the dipole formalism: They introduce an additional parameter $\alpha$ and constrain the phase space over which the dipole factors are subtracted:

$$
d \sigma^{A}=\sum_{\text {dipoles }} d \sigma^{B} \otimes d V_{\text {dipole }} \Theta\left(y_{i j, k}<\alpha\right)
$$


where the dimensionless variable $y_{i j, k}$ is given by

$$
y_{i j, k}=\frac{p_{i} p_{j}}{p_{i} p_{j}+p_{j} p_{k}+p_{i} p_{k}}
$$

The three methods (remapping of phase space, color decomposition and restriction of the phase space for the dipole factors) could be combined, if there is any need for further improvement in the efficiency.

\section{$3 \quad$ Regularization Schemes and Splitting Functions}

Theoretical calculations of infrared-safe quantities in QCD should lead to unambiguous results, independent of the chosen regularization scheme. Terms sensitive to the precise definition of the regularization scheme enter the calculation usually in the virtual part through tensor loop integrals, and in the real emission part through the splitting functions [12, which enter the dipole factors (in the dipole formalism) or the contribution from unresolved phase space ( within phase space slicing). Within dimensional regularization the most commonly used schemes are the conventional dimensional regularization scheme (CDR) [13, where all momenta and all polarization vectors are taken to be in $D$ dimensions, the 't Hooft-Veltman scheme (HV) [14], where the momenta and the helicities of the unobserved particles are $D$ dimensional, whereas the momenta and the helicities of the observed particles are 4 dimensional, and the four-dimensional helicity scheme (FDH) [15], where all polarization vectors are kept in four dimensions, as well as the momenta of the observed particles. Only the momenta of the unobserved particles are continued to $D$ dimensions.

The procedure adopted in the numerical program is as follows. The one-loop amplitudes, calculated in the four-dimensional helicity scheme, were converted to the HV scheme, using the transition rules [16],

$$
A_{F D H}^{\text {one-loop }}-A_{H V}^{\text {one-loop }}=c_{\Gamma} g^{2} A^{\text {tree }}\left(\frac{N_{c}}{3}-\frac{n_{q}}{4 N_{c}}+\frac{n_{q} N_{c}}{12}\right)
$$

where $n_{q}$ is the number of quarks. The one-loop amplitude for $e^{+} e^{-} \rightarrow q \bar{q} g g$ is converted into the 't Hooft-Veltman scheme by

$$
A_{H V}^{\text {one-loop }}=A_{F D H}^{\text {one-loop }}-c_{\Gamma} \frac{1}{2} g^{2} N_{C}\left(1-\frac{1}{N_{C}^{2}}\right) A^{\text {tree }}
$$

whereas the one-loop amplitude for $e^{+} e^{-} \rightarrow q \bar{q} q^{\prime} \bar{q}^{\prime}$ is converted by

$$
A_{H V}^{\text {one-loop }}=A_{F D H}^{\text {one-loop }}-c_{\Gamma} g^{2} N_{C}\left(\frac{2}{3}-\frac{1}{N_{C}^{2}}\right) A^{\text {tree }} .
$$

The splitting functions entering the contribution from the integrals over the dipole factors have then to be taken in the 't Hooft-Veltman scheme as well. We take the formulæ for the splitting functions in the HV-scheme from Catani, Seymour and Trócsányi [17]. They 
are given by

$$
\begin{aligned}
& P_{g \rightarrow g g}=2 C_{A}\left(\frac{z}{1-z}+\frac{1-z}{z}+z(1-z)\right), \\
& P_{q \rightarrow q g}=2 C_{F}\left(\frac{2 z}{1-z}+(1-\varepsilon)(1-z)\right), \\
& P_{g \rightarrow q \bar{q}}=2 T_{R} N_{f}\left(1-\frac{2}{1-\varepsilon} z(1-z)\right)
\end{aligned}
$$

A statistical factor of $1 / 2$ ! for two identical particles is included in the $g \rightarrow g g$ case.

\section{Phenomenology}

The numerical program MERCUTIO developed in this work is a general purpose program for calculating any infrared safe four-, three- and two-jet observable to next-to-leading order in $\alpha_{s}$ (and five-jet quantities at leading order). It is written in the language $\mathrm{C}++$. The only approximations which have been made are the neglect of the light quark masses and terms which are suppressed by $1 / m_{\text {top }}^{4}$ or higher powers of the top quark mass. MERCUTIO calculates the quantities in fixed order in $\alpha_{s}$, no resummation of terms of $\ln ^{2} y_{\text {cut }}$ or $\ln y_{\text {cut }}$ has (yet) been implemented. Therefore our results are reliable only for values of $y_{\text {cut }}$ which are not too small. The program calculates the jet quantities at the partonic level, and no hadronization is done.

The distribution for an infrared-safe variable $O$ at the center-of-mass energy $Q$ at nextto-leading order is given by two coefficients, $B_{O}$ and $C_{O}$, which represent the leading and next-to-leading order perturbative contributions:

$$
\frac{1}{\sigma_{t o t}} O \frac{d \sigma}{d O}=\left(\frac{\alpha_{s}}{2 \pi}\right)^{2} B_{O}+\left(\frac{\alpha_{s}}{2 \pi}\right)^{3}\left(C_{O}+2 \beta_{0} \ln \left(\frac{\mu^{2}}{Q^{2}}\right) B_{O}\right)
$$

We normalize all distributions to the total hadronic cross section $\sigma_{\text {tot }}$ at $O\left(\alpha_{s}\right)$, given by

$$
\sigma_{t o t}=\sigma_{2-j e t}^{\text {Born }}\left(1+\frac{\alpha_{s}}{\pi}\right)
$$

We further normalize each variable such that it takes values between 0 and 1 . The average of the variable is then defined as

$$
\langle O\rangle=\frac{1}{\sigma_{t o t}} \int_{0}^{1} O \frac{d \sigma}{d O} d O .
$$

As our nominal choice of input parameters we use $N_{c}=3$ colors and $N_{f}=5$ massless quarks. We take the electromagnetic coupling to be $\alpha\left(m_{Z}\right)=1 / 127.9$ and the strong coupling to be $\alpha_{s}\left(m_{Z}\right)=0.118$. The numerical values of the $Z^{0}$-mass and width are $m_{Z}=91.187 \mathrm{GeV}$ and $\Gamma_{Z}=2.490 \mathrm{GeV}$. For the top mass we take $m_{t}=174 \mathrm{GeV}$ and for the weak mixing angle $\sin ^{2} \theta_{W}=0.230$. We take the center of mass energy to be $\sqrt{Q^{2}}=m_{Z}$ and we set the renormalization scale equal to $\mu^{2}=Q^{2}$. 


\begin{tabular}{|c|c|c|c|}
\hline Algorithm & $y_{\text {cut }}$ & MERCUTIO & MENLO PARC \\
\hline & 0.005 & $(3.93 \pm 0.02) \cdot 10^{-1}$ & $(3.79 \pm 0.08) \cdot 10^{-1}$ \\
JADE-E0 & 0.01 & $(1.93 \pm 0.01) \cdot 10^{-1}$ & $(1.88 \pm 0.03) \cdot 10^{-1}$ \\
& 0.03 & $(3.38 \pm 0.01) \cdot 10^{-2}$ & $(3.46 \pm 0.05) \cdot 10^{-2}$ \\
\hline & $y_{\text {cut }}$ & DEBRECEN & EERAD2 \\
\hline & 0.005 & $(3.88 \pm 0.07) \cdot 10^{-1}$ & $(3.87 \pm 0.03) \cdot 10^{-1}$ \\
& 0.01 & $(1.92 \pm 0.01) \cdot 10^{-1}$ & $(1.93 \pm 0.01) \cdot 10^{-1}$ \\
& 0.03 & $(3.37 \pm 0.01) \cdot 10^{-2}$ & $(3.35 \pm 0.01) \cdot 10^{-2}$ \\
\hline & & & \\
\hline Algorithm & $y_{\text {cut }}$ & MERCUTIO & MENLO PARC \\
\hline \multirow{3}{*}{ DURHAM } & 0.005 & $(1.06 \pm 0.01) \cdot 10^{-1}$ & $(1.04 \pm 0.02) \cdot 10^{-1}$ \\
& 0.01 & $(4.72 \pm 0.01) \cdot 10^{-2}$ & $(4.70 \pm 0.06) \cdot 10^{-2}$ \\
& 0.03 & $(6.96 \pm 0.03) \cdot 10^{-3}$ & $(6.82 \pm 0.08) \cdot 10^{-3}$ \\
\hline & $y_{\text {cut }}$ & DEBRECEN & EERAD2 \\
\hline & 0.005 & $(1.05 \pm 0.01) \cdot 10^{-1}$ & $(1.05 \pm 0.01) \cdot 10^{-1}$ \\
& 0.01 & $(4.66 \pm 0.02) \cdot 10^{-2}$ & $(4.65 \pm 0.02) \cdot 10^{-2}$ \\
& 0.03 & $(6.87 \pm 0.04) \cdot 10^{-3}$ & $(6.86 \pm 0.03) \cdot 10^{-3}$ \\
\hline & & & \\
\hline Algorithm & $y_{\text {cut }}$ & MERCUTIO & MENLO PARC \\
\hline \multirow{3}{*}{ GENEVA } & 0.02 & $(2.67 \pm 0.05) \cdot 10^{-1}$ & $(2.56 \pm 0.06) \cdot 10^{-1}$ \\
& 0.03 & $(1.79 \pm 0.03) \cdot 10^{-1}$ & $(1.71 \pm 0.03) \cdot 10^{-1}$ \\
& 0.05 & $(8.53 \pm 0.07) \cdot 10^{-2}$ & $(8.58 \pm 0.15) \cdot 10^{-2}$ \\
\hline & $y_{\text {cut }}$ & DEBRECEN & EERAD2 \\
\hline & 0.02 & $(2.63 \pm 0.06) \cdot 10^{-1}$ & $(2.61 \pm 0.05) \cdot 10^{-1}$ \\
& 0.03 & $(1.75 \pm 0.03) \cdot 10^{-1}$ & $(1.72 \pm 0.03) \cdot 10^{-1}$ \\
& 0.05 & $(8.37 \pm 0.12) \cdot 10^{-2}$ & $(8.50 \pm 0.06) \cdot 10^{-2}$ \\
\hline
\end{tabular}

Table 1: The four-jet fraction as calculated by Mercutio, MENLO PARC, DEBRECEN and EERAD2, for different jet algorithms and varying $y_{\text {cut }}$.

\subsection{Four-Jet Fraction}

The four-jet fraction has been calculated by each group which has provided a numerical NLO four-jet program and serves as a cross-check. The four-jet fraction is defined as

$$
R_{4}=\frac{\sigma_{4-j e t}}{\sigma_{t o t}} .
$$

The values obtained for the four-jet fraction for different jet algorithms ( JADE [18], DURHAM [19] and GENEVA [20] ) and varying $y_{\text {cut }}$ are given in table 1, together with the corresponding values from the programs MENLO PARC by L. Dixon and A. Signer [3], DEBRECEN by Z. Nagy and Z. Trócsányi [4] and EERAD2 by J.M. Campbell, M.A. Cullen and E.W.N. Glover [5]. 


\subsection{The D-Parameter}

Global event shape variables describe the topology of an event. They may be calculated without reference to a jet defining algorithm. Like jet algorithms they have to be infrared safe. An example of a global event shape variable is the $D$-parameter [21] which is derived from the eigenvalues of the momentum tensor

$$
\theta^{i j}=\frac{\sum_{a} \frac{p_{a}^{i} p_{a}^{j}}{\left|\vec{p}_{a}\right|}}{\sum_{a}\left|\vec{p}_{a}\right|}
$$

where the sum runs over all final state particles and $p_{a}^{i}$ is the $i$-th component of the threemomentum $\vec{p}_{a}$ of particle $a$ in the c.m. system. The tensor $\theta$ is normalized to have unit trace. In terms of the eigenvalues of the $\theta$ tensor, $\lambda_{1}, \lambda_{2}, \lambda_{3}$, with $\lambda_{1}+\lambda_{2}+\lambda_{3}=1$, one defines

$$
D=27 \lambda_{1} \lambda_{2} \lambda_{3}=27 \operatorname{det} \theta^{i j}
$$

The range of values is $0 \leq D \leq 1$. The $D$-parameter measures aplanarity, since one needs at least four final-state particles to obtain a non-vanishing value.

The values for the functions $B_{D}$ and $C_{D}$ are given in table 2. Figure 1 shows the Dparameter distribution. We remark that we have chosen to normalize the distribution to the total hadronic cross-section at $O\left(\alpha_{s}\right)$. The average of the shape variable is defined as

$$
\langle D\rangle=\frac{1}{\sigma_{t o t}} \int_{0}^{1} D \frac{d \sigma}{d D} d D .
$$

For the average we obtain

$$
\langle D\rangle=\left(\frac{\alpha_{s}}{2 \pi}\right)^{2}(5.82 \pm 0.01) \cdot 10^{1}+\left(\frac{\alpha_{s}}{2 \pi}\right)^{3}(2.43 \pm 0.06) \cdot 10^{3} .
$$

These numbers agree with the ones given by Nagy and Trócsányi as published in ref. [4] after taking care of different normalizations ( $\sigma_{\text {tot }}$ in our case and $\sigma_{2-\text { jet }}^{\text {Born }}$ in ref. 四). The $D$-parameter distribution has also been calculated by Campbell, Cullen and Glover [5].

\subsection{The Jet Broadening Variable}

With the numerical program for $e^{+} e^{-} \rightarrow 4$ jets one may also study the internal structure of three-jets events. One example is the jet broadening variable defined as [22]

$$
B_{\text {jet }}=\frac{1}{n_{\text {jets }}} \sum_{\text {jets }} \frac{\sum_{a}\left|p_{a}^{\perp}\right|}{\sum_{a}\left|\vec{p}_{a}\right|}
$$

Here $p_{a}^{\perp}$ is the momentum of particle $a$ transverse to the jet axis of jet $J$, and the sum over $a$ extends over all particles in the jet $J$. The jet broadening variable $B_{\text {jet }}$ considered here shall not be confused with the narrow jet broadening $B_{\text {min }}$, which is defined differently. The later one has been recently calculated to NLO by Campbell, Cullen and Glover [5]. 


\begin{tabular}{|c|cc|}
\hline$D$ & $B_{D}$ & $C_{D}$ \\
\hline 0.02 & $(6.36 \pm 0.04) \cdot 10^{2}$ & $(1.05 \pm 0.13) \cdot 10^{4}$ \\
0.06 & $(2.24 \pm 0.01) \cdot 10^{2}$ & $(1.21 \pm 0.03) \cdot 10^{4}$ \\
0.10 & $(1.41 \pm 0.01) \cdot 10^{2}$ & $(8.37 \pm 0.26) \cdot 10^{3}$ \\
0.14 & $(9.96 \pm 0.08) \cdot 10^{1}$ & $(6.19 \pm 0.26) \cdot 10^{3}$ \\
0.18 & $(7.43 \pm 0.04) \cdot 10^{1}$ & $(4.84 \pm 0.25) \cdot 10^{3}$ \\
0.22 & $(5.74 \pm 0.03) \cdot 10^{1}$ & $(3.76 \pm 0.08) \cdot 10^{3}$ \\
0.26 & $(4.51 \pm 0.04) \cdot 10^{1}$ & $(3.00 \pm 0.13) \cdot 10^{3}$ \\
0.30 & $(3.61 \pm 0.02) \cdot 10^{1}$ & $(2.43 \pm 0.09) \cdot 10^{3}$ \\
0.34 & $(2.90 \pm 0.02) \cdot 10^{1}$ & $(1.93 \pm 0.19) \cdot 10^{3}$ \\
0.38 & $(2.33 \pm 0.02) \cdot 10^{1}$ & $(1.56 \pm 0.11) \cdot 10^{3}$ \\
0.42 & $(1.91 \pm 0.03) \cdot 10^{1}$ & $(1.29 \pm 0.11) \cdot 10^{3}$ \\
0.46 & $(1.55 \pm 0.01) \cdot 10^{1}$ & $(1.06 \pm 0.10) \cdot 10^{3}$ \\
0.50 & $(1.26 \pm 0.02) \cdot 10^{1}$ & $(8.08 \pm 0.93) \cdot 10^{2}$ \\
0.54 & $(1.02 \pm 0.02) \cdot 10^{1}$ & $(7.10 \pm 0.72) \cdot 10^{2}$ \\
0.58 & $(8.20 \pm 0.16) \cdot 10^{0}$ & $(5.64 \pm 0.64) \cdot 10^{2}$ \\
0.62 & $(6.52 \pm 0.12) \cdot 10^{0}$ & $(4.27 \pm 0.37) \cdot 10^{2}$ \\
0.66 & $(5.09 \pm 0.08) \cdot 10^{0}$ & $(3.32 \pm 0.46) \cdot 10^{2}$ \\
0.70 & $(3.97 \pm 0.05) \cdot 10^{0}$ & $(2.68 \pm 0.35) \cdot 10^{2}$ \\
0.74 & $(2.94 \pm 0.06) \cdot 10^{0}$ & $(2.19 \pm 0.35) \cdot 10^{2}$ \\
0.78 & $(2.15 \pm 0.08) \cdot 10^{0}$ & $(1.34 \pm 0.15) \cdot 10^{2}$ \\
0.82 & $(1.52 \pm 0.05) \cdot 10^{0}$ & $(1.14 \pm 0.20) \cdot 10^{2}$ \\
0.86 & $(9.82 \pm 0.52) \cdot 10^{-1}$ & $(6.28 \pm 1.15) \cdot 10^{1}$ \\
0.90 & $(5.44 \pm 0.46) \cdot 10^{-1}$ & $(3.55 \pm 0.79) \cdot 10^{1}$ \\
0.94 & $(2.38 \pm 0.10) \cdot 10^{-1}$ & $(1.87 \pm 1.03) \cdot 10^{1}$ \\
0.98 & $(4.70 \pm 1.09) \cdot 10^{-2}$ & $(2.15 \pm 8.31) \cdot 10^{0}$ \\
\hline
\end{tabular}

Table 2: The Born level and next-to-leading order functions $B_{D}$ and $C_{D}$ for the $D$ parameter. 


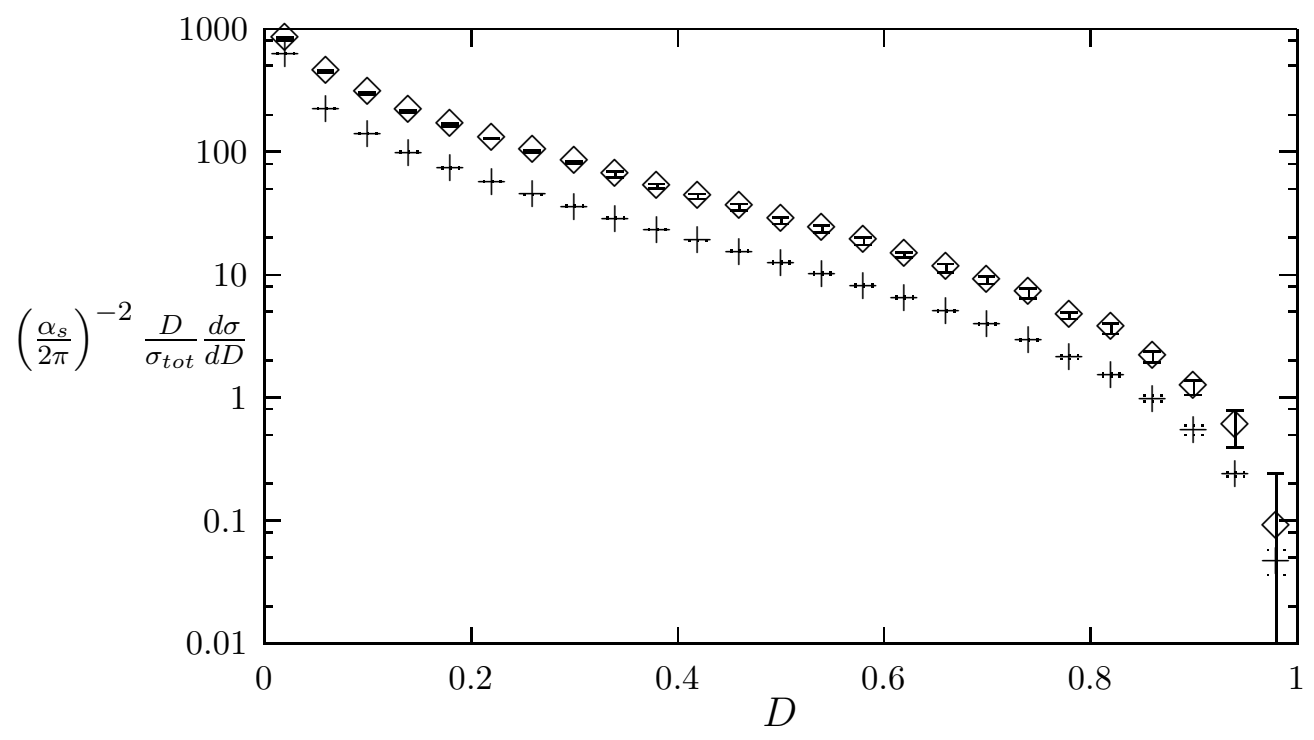

Figure 1: The D-parameter distribution at NLO (diamonds) and LO (crosses).

The jet broadening variable is calculated for three-jet events defined by the DURHAM algorithm and $y_{c u t}=0.1$. This choice is motivated by a recent analysis of the Aleph collaboration [22]. For the average we obtain

$$
\left\langle B_{j e t}\right\rangle=\left(\frac{\alpha_{s}}{2 \pi}\right)^{2}(1.79 \pm 0.01) \cdot 10^{1}+\left(\frac{\alpha_{s}}{2 \pi}\right)^{3}(4.87 \pm 0.31) \cdot 10^{2} .
$$

The values for the functions $B_{B_{j e t}}$ and $C_{B_{j e t}}$ are given in table 3 . Figure 2 shows the distribution of the jet broadening variable.

\subsection{The Softest-jet Explanarity}

For three jet events we define the softest-jet explanarity as follows:

$$
E_{3 j e t}=\frac{\sum_{a}\left|\vec{p}_{a} \cdot\left(\vec{P}_{1} \times \vec{P}_{2}\right)\right|}{\sum_{a}\left|\vec{p}_{a}\right|\left|\vec{P}_{1} \times \vec{P}_{2}\right|}
$$

where $\vec{p}_{a}$ is the three-momentum of particle $a$ inside the softest jet and the sum runs over all particles in the softest jet. $\vec{P}_{1}$ and $\vec{P}_{2}$ denote the three-momenta of the remaining two jets in the event. The explanarity measures the degree to which the shape of the softest jet lies out of the event plane. The definition is motivated by the fact, that the explanarity will be sensitive to the color factors of QCD.

The softest-jet explanarity is calculated for three-jet events defined by the DURHAM algorithm and $y_{c u t}=0.1$. For the average we obtain

$$
\left\langle E_{3 j e t}\right\rangle=\left(\frac{\alpha_{s}}{2 \pi}\right)^{2}(1.29 \pm 0.01) \cdot 10^{1}+\left(\frac{\alpha_{s}}{2 \pi}\right)^{3}(3.33 \pm 0.22) \cdot 10^{2} .
$$




\begin{tabular}{|c|cc|}
\hline$B_{\text {jet }}$ & $B_{B_{\text {jet }}}$ & $C_{B_{\text {jet }}}$ \\
\hline 0.02 & $(7.31 \pm 0.22) \cdot 10^{1}$ & $(-1.71 \pm 0.66) \cdot 10^{3}$ \\
0.06 & $(5.13 \pm 0.05) \cdot 10^{1}$ & $(9.23 \pm 2.79) \cdot 10^{2}$ \\
0.10 & $(4.27 \pm 0.06) \cdot 10^{1}$ & $(1.27 \pm 0.20) \cdot 10^{3}$ \\
0.14 & $(3.75 \pm 0.06) \cdot 10^{1}$ & $(1.24 \pm 0.16) \cdot 10^{3}$ \\
0.18 & $(3.36 \pm 0.06) \cdot 10^{1}$ & $(1.26 \pm 0.14) \cdot 10^{3}$ \\
0.22 & $(2.99 \pm 0.03) \cdot 10^{1}$ & $(1.28 \pm 0.14) \cdot 10^{3}$ \\
0.26 & $(2.74 \pm 0.03) \cdot 10^{1}$ & $(1.13 \pm 0.12) \cdot 10^{3}$ \\
0.30 & $(2.50 \pm 0.02) \cdot 10^{1}$ & $(1.06 \pm 0.07) \cdot 10^{3}$ \\
0.34 & $(2.28 \pm 0.03) \cdot 10^{1}$ & $(1.00 \pm 0.07) \cdot 10^{3}$ \\
0.38 & $(2.08 \pm 0.02) \cdot 10^{1}$ & $(9.12 \pm 0.51) \cdot 10^{2}$ \\
0.42 & $(1.87 \pm 0.01) \cdot 10^{1}$ & $(7.92 \pm 0.69) \cdot 10^{2}$ \\
0.46 & $(1.67 \pm 0.02) \cdot 10^{1}$ & $(7.59 \pm 1.19) \cdot 10^{2}$ \\
0.50 & $(1.44 \pm 0.02) \cdot 10^{1}$ & $(6.36 \pm 1.43) \cdot 10^{2}$ \\
0.54 & $(1.20 \pm 0.01) \cdot 10^{1}$ & $(5.75 \pm 0.30) \cdot 10^{2}$ \\
0.58 & $(9.42 \pm 0.18) \cdot 10^{0}$ & $(4.30 \pm 0.33) \cdot 10^{2}$ \\
0.62 & $(6.46 \pm 0.12) \cdot 10^{0}$ & $(3.33 \pm 0.65) \cdot 10^{2}$ \\
0.66 & $(3.76 \pm 0.07) \cdot 10^{0}$ & $(1.84 \pm 0.18) \cdot 10^{2}$ \\
0.70 & $(1.60 \pm 0.04) \cdot 10^{0}$ & $(7.97 \pm 1.07) \cdot 10^{1}$ \\
0.74 & $(3.39 \pm 0.28) \cdot 10^{-1}$ & $(1.95 \pm 0.55) \cdot 10^{1}$ \\
0.78 & $(1.11 \pm 0.31) \cdot 10^{-2}$ & $(2.79 \pm 1.24) \cdot 10^{0}$ \\
0.82 & 0.00 & $(8.26 \pm 9.73) \cdot 10^{-2}$ \\
0.86 & 0.00 & 0.00 \\
0.90 & 0.00 & 0.00 \\
0.94 & 0.00 & 0.00 \\
0.98 & 0.00 & 0.00 \\
\hline
\end{tabular}

Table 3: The Born level and next-to-leading order functions $B_{B_{j e t}}$ and $C_{B_{j e t}}$ for the jet broadening variable. 


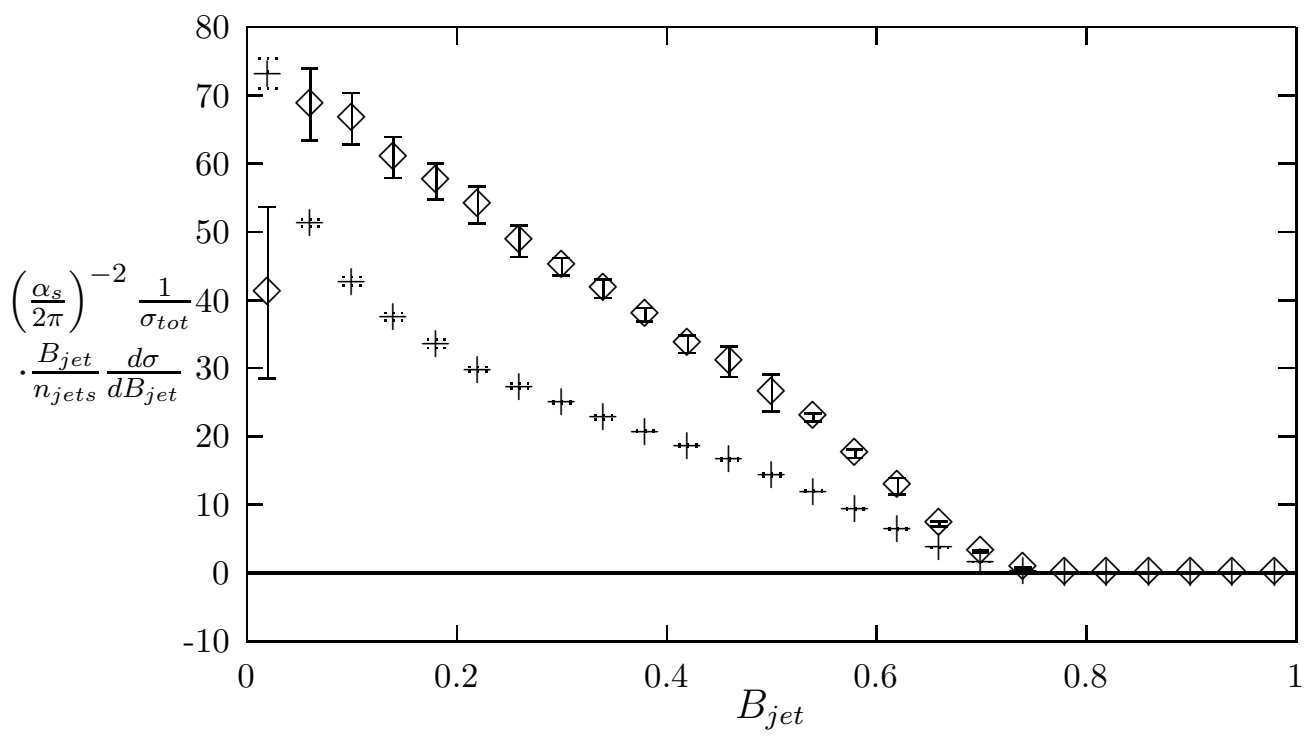

Figure 2: The $B_{\text {jet }}$ distribution at NLO (diamonds) and LO (crosses).

The values for the functions $B_{E_{3 j e t}}$ and $C_{E_{3 j e t}}$ are given in table 4 . Figure 3 shows the distribution of the softest-jet explanarity.

\section{Conclusions}

In this paper we reported on the general purpose numerical program "MERCUTIO", which can be used to calculate any infrared safe four-jet quantity in electron-positron annihilation at next-to-leading order. The program is based on the dipole formalism and uses a remapping of phase-space in order to improve the efficiency of the Monte Carlo integration. We presented numerical results for the four-jet fraction and the $D$-parameter. These results agree with the results from other groups. The program can also be used to investigate the internal structure of three-jet events at NLO. We also presented results for previously uncalculated observables: the jet broadening variable and the softest-jet explanarity.

\section{References}

[1] Z. Bern, L. Dixon, D. A. Kosower, and S. Weinzierl, Nucl. Phys. B489, (1997), 3

Z. Bern, L. Dixon and D. A. Kosower, Nucl. Phys. B513, (1998), 3

[2] E. W. N. Glover and D. J. Miller, Phys. Lett. B396, (1997), 257

J. M. Campbell, E. W. N. Glover, and D. J. Miller, Phys. Lett. B409, (1997), 503

[3] L. Dixon and A. Signer, Phys. Rev. Lett. 78, (1997), 811

A. Signer, hep-ph/9705218

L. Dixon and A. Signer, Phys. Rev. D56, (1997), 4031 


\begin{tabular}{|c|cc|}
\hline$E_{3 j e t}$ & $B_{E_{3 j e t}}$ & $C_{E_{3 j e t}}$ \\
\hline 0.02 & $(7.06 \pm 0.16) \cdot 10^{1}$ & $(-1.26 \pm 0.58) \cdot 10^{3}$ \\
0.06 & $(4.59 \pm 0.07) \cdot 10^{1}$ & $(1.30 \pm 0.13) \cdot 10^{3}$ \\
0.10 & $(3.61 \pm 0.04) \cdot 10^{1}$ & $(1.35 \pm 0.16) \cdot 10^{3}$ \\
0.14 & $(2.99 \pm 0.02) \cdot 10^{1}$ & $(1.19 \pm 0.12) \cdot 10^{3}$ \\
0.18 & $(2.51 \pm 0.02) \cdot 10^{1}$ & $(1.02 \pm 0.11) \cdot 10^{3}$ \\
0.22 & $(2.13 \pm 0.02) \cdot 10^{1}$ & $(9.65 \pm 0.86) \cdot 10^{2}$ \\
0.26 & $(1.83 \pm 0.01) \cdot 10^{1}$ & $(8.13 \pm 1.37) \cdot 10^{2}$ \\
0.30 & $(1.55 \pm 0.02) \cdot 10^{1}$ & $(6.68 \pm 0.72) \cdot 10^{2}$ \\
0.34 & $(1.32 \pm 0.02) \cdot 10^{1}$ & $(5.28 \pm 0.60) \cdot 10^{2}$ \\
0.38 & $(1.12 \pm 0.01) \cdot 10^{1}$ & $(4.88 \pm 0.45) \cdot 10^{2}$ \\
0.42 & $(9.32 \pm 0.13) \cdot 10^{0}$ & $(3.70 \pm 0.53) \cdot 10^{2}$ \\
0.46 & $(7.62 \pm 0.10) \cdot 10^{0}$ & $(3.14 \pm 0.33) \cdot 10^{2}$ \\
0.50 & $(6.07 \pm 0.15) \cdot 10^{0}$ & $(2.03 \pm 0.34) \cdot 10^{2}$ \\
0.54 & $(4.61 \pm 0.10) \cdot 10^{0}$ & $(1.70 \pm 0.32) \cdot 10^{2}$ \\
0.58 & $(3.45 \pm 0.07) \cdot 10^{0}$ & $(1.15 \pm 0.50) \cdot 10^{2}$ \\
0.62 & $(2.26 \pm 0.09) \cdot 10^{0}$ & $(5.97 \pm 2.68) \cdot 10^{1}$ \\
0.66 & $(1.28 \pm 0.04) \cdot 10^{0}$ & $(2.73 \pm 1.82) \cdot 10^{1}$ \\
0.70 & $(5.04 \pm 0.20) \cdot 10^{-1}$ & $(9.87 \pm 8.67) \cdot 10^{0}$ \\
0.74 & $(7.20 \pm 1.16) \cdot 10^{-2}$ & $(-0.70 \pm 3.08) \cdot 10^{0}$ \\
0.78 & $(2.07 \pm 2.68) \cdot 10^{-4}$ & $(-2.04 \pm 2.80) \cdot 10^{-2}$ \\
0.82 & 0.00 & 0.00 \\
0.86 & 0.00 & 0.00 \\
0.90 & 0.00 & 0.00 \\
0.94 & 0.00 & 0.00 \\
0.98 & 0.00 & 0.00 \\
\hline
\end{tabular}

Table 4: The Born level and next-to-leading order functions $B_{E_{3 j e t}}$ and $C_{E_{3 j e t}}$ for the softest-jet explanarity. 


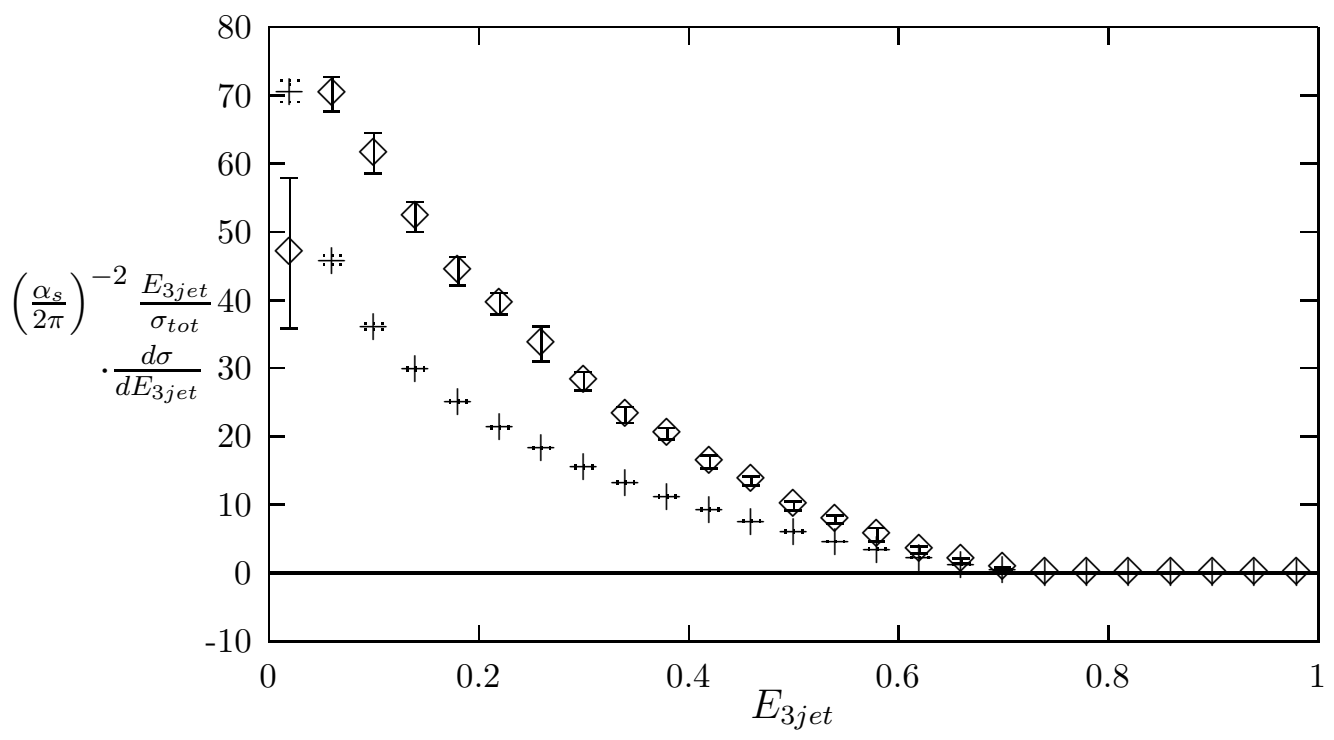

Figure 3: The $E_{3 j e t}$ distribution at NLO (diamonds) and LO (crosses).

[4] Z. Nagy and Z. Trócsányi, Nucl. Phys. B486, (1997), 189

Z. Nagy and Z. Trócsányi, Phys. Rev. Lett 79, (1997), 3604

Z. Nagy and Z. Trócsányi, Phys. Lett. B414, (1997), 187

Z. Nagy and Z. Trócsányi, hep-ph/9708343

Z. Nagy and Z. Trócsányi, Nucl. Phys. Proc. Suppl. 64, (1998), 63

Z. Nagy and Z. Trócsányi, Phys. Rev. D57, (1998), 5793

Z. Nagy and Z. Trócsányi, Phys. Rev. D59, (1999), 014020

Z. Nagy and Z. Trócsányi, hep-ph/9808364

[5] J. M. Campbell, M. A. Cullen, and E. W. N. Glover, hep-ph/9809429

[6] S. Frixione, Z. Kunszt, and A. Signer, Nucl. Phys. B467, (1996), 399

[7] S. Catani, M. H. Seymour, Nucl. Phys. B485, (1997), 291, Erratum-ibid. B510, (1997), 503,

[8] W. L. van Neerven and J. A. M. Vermaseren, Nucl. Phys. B238, (1984), 73;

H. Baer, J. Ohnemus, and J. F. Owens, Phys. Rev. D40:2844 (1989);

K. Fabricius, G. Kramer, G. Schierholz, and I. Schmitt, Z. Phys. C11:315 (1981);

G. Kramer and B. Lampe, Fortschr. Phys. 37:161 (1989)

[9] W. T. Giele and E. W. N. Glover, Phys. Rev. D 46, (1992), 1980

[10] F. A. Berends, W. T. Giele, and H. Kuijf, Nucl. Phys. B321, (1989), 39

[11] Z. Bern, L. Dixon, D. C. Dunbar, and D. A. Kosower, Nucl. Phys. B425, (1994), 217

[12] G. Altarelli and G. Parisi, Nucl. Phys. B126, (1977), 298

[13] J. C. Collins, Renormalization, Cambridge University Press, 1984 
[14] G. 't Hooft and M. Veltman, Nucl. Phys. B44, (1972), 189

[15] Z. Bern and D. A. Kosower, Nucl. Phys. B379, (1992), 451

[16] A. Signer, Ph. D. thesis, Diss. ETH Nr. 11143

[17] S. Catani, M. H. Seymour, and Z. Trócsányi, Phys. Rev. D55, (1997), 6819

[18] W. Bartel et al. , Z. Phys. C 33, (1986), 23

[19] Report of the Hard QCD Working Group, in Proc. Durham Workshop on Jet Studies at LEP and HERA, J. Phys. G17, (1991), 1537

[20] S. Bethke, Z. Kunszt, D. E. Soper, and W. J. Stirling, Nucl. Phys B370, (1992), 310

[21] G. Parisi, Phys. Lett. 74B, (1978), 65

[22] R. Barate et al., Aleph Collaboration, CERN-EP/98-16 